THE EFFECT OF FINITE BOUNDARIES

ON THE MOTION OF PARTICLES IN NON-NEWTONIAN FLUIDS - I

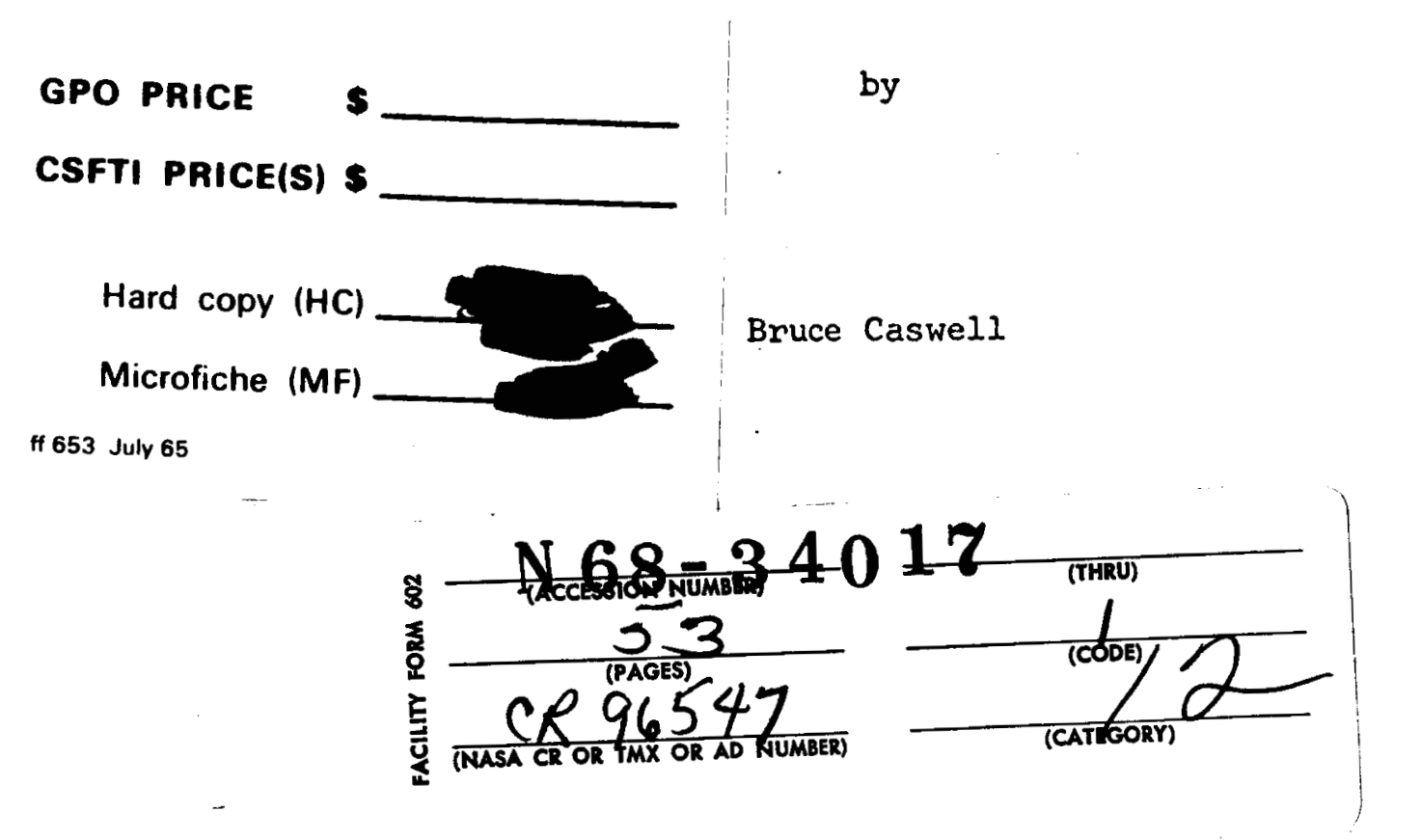

Division of Engineering.

and Center for Fluid Dynamics

BROWN UNIVERSITY

Providence, Rhode Island
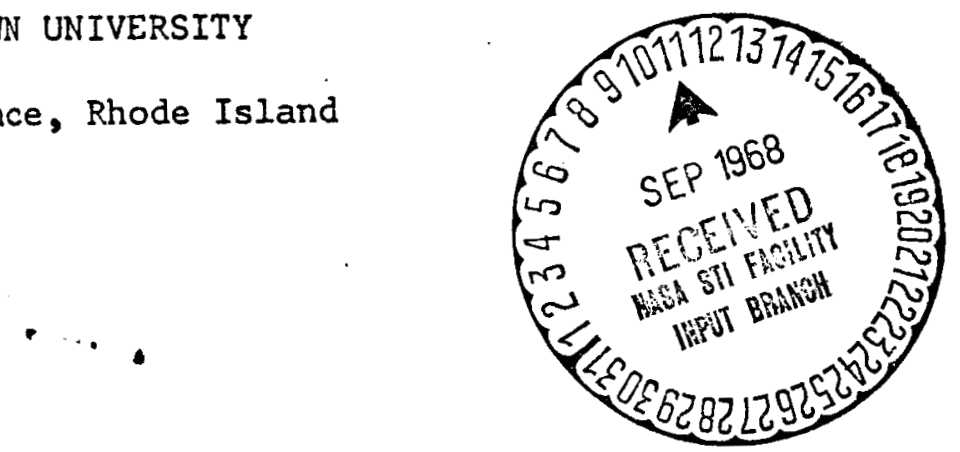

June $\mathbf{1 9 6 8}$ 
1. Introduction

The effect of container boundaries on the Stokes resistance of a particle moving in a viscoelastic fluid has been found as a correction to the velocity $\left(\bar{U}_{\infty}, \bar{\Omega}_{\infty}\right)$ in an infinite region, and is given by

$$
\begin{aligned}
& \bar{U}=\bar{U}_{\infty}+\bar{k} \cdot \bar{F} / 6 \pi \mu_{0} l+o\left(l^{-2}\right), \\
& \bar{\Omega}=\bar{\Omega}_{\infty}+o\left(l^{-2}\right) .
\end{aligned}
$$

Here $\bar{U}$ and $\bar{\Omega}$ are respectively the translational and rotational velocities of the particle under the influence of an outside force $-\bar{F}$ and outside torque $-\bar{L}$ when located at a distance $l$ from a boundary whose wall-effect tensor (Brenner [1964a]) is $\bar{k}$. Because of the non-linearity of the fluid's constitutive relation the velocities $\left(\bar{U}_{\infty}, \bar{\Omega}_{\infty}\right)$ with which the particle would move in an unbounded domain under the influence of the same force and torque are given by non-linear equations

$$
\bar{U}_{\infty}=\bar{U}_{\infty}(\bar{F}, \bar{L}), \bar{\Omega}_{\infty}=\bar{\Omega}_{\infty}(\bar{F}, \bar{L})
$$

Hence, in general, it is not possible to conveniently express the wall effect as a force correction formula. In the Newtonian case the linearity of (1.2) renders the inversion of (1.1) a trivial calculation, and in fact Brenner [1964a] first obtained this formula by inversion of his force correction formula valid for Newtonian fluids only. The only restriction on the constitutive equation for the validity of (1.1) is that it must describe an isotropic fluid which has a lower Newtonian regime with zero-shear viscosity $\mu_{0}$.

When the outer boundary possesses a high degree of symmetry the error estimate in $(1.1)$ is reduced to $O\left(l^{-3}\right)$. Several cases of experimental interest 
fall into this category. Explicit formulae for the terms of $O\left(l^{-2}\right)$ are given, and from these the rotational stability of sedimenting particles can be deduced. In particular, it is shown why the edgewise position is the stable orientation for a disk sedimenting in a circular cylinder. It is also possible to explain the radial migration, observed by Tanner (1964), for spheres falling along the axis of a tube filled with a polymer solution.

\section{Constitutive Relations}

The analysis given below is based on the constitutive relation of incompressible Rivlin-Ericksen ( 1955 ) fluids which is given by

$$
\overline{\mathrm{T}}=-\overline{\overline{\mathrm{I}}} \mathrm{p}+\overline{\overline{\mathrm{f}}(\overline{\mathrm{A}}}(1), \overline{\bar{A}}(2), \ldots, \overline{\bar{A}}(\mathrm{~N}),
$$

where $\mathrm{p}$ is the scalar pressure and $\overline{\bar{f}}$ is an isotropic tensor function of the Rivlin-Ericksen tensors $\bar{A}(L)(L=1, \ldots, N)$. The tensor $\bar{A}(l)$ is twice the rate of strain tensor, and the higher order tensors are constructed from the recurrence relation

$$
\bar{A}(N)=\frac{D \bar{A}(N-1)}{D t}+\frac{1}{2}[\overline{\bar{A}}(1) \cdot \bar{A}(N-1)+\bar{A}(N-1) \cdot \bar{A}(1)] .
$$

The corotational time derivative which appears in (2.2) can be written as an operator of the form

$$
\frac{D}{D t}=\frac{D}{D t}+\frac{I}{2}[x \bar{w}-\bar{w} x \cdot],
$$

where $D / D t$ is the material derivative and $\bar{\omega}$ the vorticity. For sufficiently slow motions ${ }^{l}$ it can be shown that $\dot{f}$ can be approximated by multinomials in

I These motions are siow in the viscoelastic sense, but are not necessarily low Reynolds number flows. 
the $\overline{\bar{A}}^{(N)}$. After appropriate reduction such multinomial expansions can be ordered as follows:

$\overline{\bar{T}}^{i}=-\overline{\bar{l}}_{\mathrm{p}}+\mu_{\mathrm{o}} \overline{\bar{A}}(1) \quad$ 2st order (Newtonian),

$\overline{\mathrm{T}}^{i i}=\overline{\mathrm{T}}^{i}+\alpha_{1} \overline{\bar{A}}^{(2)}+\alpha_{2}(\overline{\bar{A}}(1))^{2} \quad$ 2nd order,

$\overline{\bar{T}} i i i=\overline{\bar{T}}^{i i}+B_{1} \overline{\bar{A}}^{(3)}+\beta_{2}\left(\overline{\bar{A}}^{(1)} \cdot \overline{\bar{A}}(2)+\overline{\bar{A}}(2) \cdot \overline{\bar{A}}^{(1)}\right)+\beta_{3}(\operatorname{tr} \overline{\bar{A}}(2)) \overline{\bar{A}}^{(1)}$ 3rd order,

etc.,

where $\mu_{0}$, the $\alpha^{\prime}$ s and the $\beta^{\prime}$ s are material constants. These slow flow approximations were first obtained by Langlois and Rivlin [1959] directly from (2.1); later Coleman and Noll [1961] were able to derive the same equations from their theory of simple fluids.

In order to substantiate the generality claimed for the principle result (1.1) we examine the position of the Rivlin-Ericksen theory within the framework of more general theories. In the first place it has been shown by Green and Rivlin [1960] that their general constitutive equation of the memory type reduces to (2.1) in well-behaved steady flows. Secondly, it has already been mentioned above that the slow flow approximations (2.4), (2.5), and (2.6) can be derived from the still more general theory of simple fluids. Lastly, within the framework of the perturbation analysis to be given below it can be shown (see the Appendix) that many of the special theories currently in vogue reduce to the same set of governing equations. The Rivlin-Ericksen theory appears to give an adequate description of the stresses in real fluids undergoing slow flows, but it is clearly at odds 
with experiment when the same fluids undergo certain other motions. Therefore, it is desirable to classify the flow which the real fluid is undergoing. This seems preferable to the practice of many authors who try to classify the fluid according to the constitutive relation. This paper deals only with Nth order viscoelastic flows. In such flows the stress is given by the Nth approximation to (2.1.). Hence (2.6) is the stress relation for a third order viscoelastic flow. The justification for the assumption of Nth order flow depends upon the relative magnitudes of characteristic times for the flow and the fluid. This is closely analogous to the assumption of isochoric flow of a compressible fiuid which is justified when the characteristic velocity of the flow is small compared to that of the fluid.

\section{The Governing Equations}

In viscoelastic flow the neglect of inertia does not lead to a linear problem as in the Newtonian case. However, the form of the slow flow approximations suggests the existence of velocity and pressure expansions of the form

$$
\begin{aligned}
& \bar{v}=\bar{v}_{1}+\bar{v}_{2}+\bar{v}_{3}+\ldots, \\
& p=p_{1}+p_{2}+p_{3}+\ldots \cdot
\end{aligned}
$$

The fields $\overline{\mathrm{V}}_{1}, \mathrm{p}_{1}$ are made to satisfy the linear Stokes equations, and the higher order fields $\bar{v}_{i}, p_{i}(i>1)$ then satisfy inhomogeneous Stokes equations. Usually a viscoelastic perturbation parameter, say $\lambda$ (see Caswell and Schwarz [1962]), is introduced by use of dimensional arguments. In this analysis it is not necessary to exhibit the perturbation parameter explicitly; however, it is useful to bear in mind that the fields $\bar{v}_{i}, p_{i}$ are $O\left(\lambda^{i-1}\right)$. 
In most boundary value problems the perturbation scheme (3.1)-(3.2) rapidly becomes complicated, and actual calculations have nearly always been terminated at the third term. The results of such calculations indicate at most the initial deviation from Newtonian behavior, and are valid for real materials over a rather narrow range of experimental conditions. However, the analysis for the wall effect does not require the fields $\bar{v}_{i}, p_{i}$ to be solved for in complete detail; hence as many terms as may be needed can be included in (3.1) and (3.2). Thus the analysis rests on the assumption that the viscoelastic fluid is undergoing Nth order flow, and that the regular expansions (3.1) and (3.2) exist.

The inhomogeneous Stokes equations governing the fields $\bar{v}_{i}, p_{i}$ can be written in the form

$$
0=-\nabla p_{i}+\mu_{0} \nabla^{2} \bar{v}_{i}+\nabla \cdot \overline{\bar{\tau}}_{i}, \quad i=1,2,3, \ldots,
$$

where $\overline{\bar{\tau}}_{i}$ is called the inhomogeneous stress tensor. From the stress approximations $(2.4),(2.5)$ and $(2.6)$ the first few $\overline{\bar{\tau}}_{i}$ can be writien down,

$$
\begin{aligned}
& \overline{\bar{\tau}}_{1}=0, \quad \overline{\bar{\tau}}_{2}=\alpha_{1} \overline{\bar{A}}^{(2)}(1,1)+\alpha_{2}\left(\overline{\bar{A}}^{(1)}(1)\right)^{2} \text {, }
\end{aligned}
$$

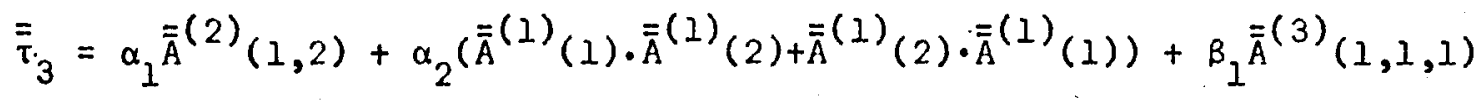

$$
\begin{aligned}
& +B_{2}\left(\overline{\bar{A}}^{(1)}(1) \cdot \overline{\bar{A}}^{(2)}(1,1)+\overline{\bar{A}}^{(2)}(1,1) \cdot \overline{\bar{A}}^{(1)}(1)\right)
\end{aligned}
$$

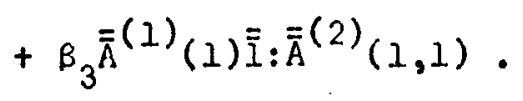

The notation $\overline{\bar{A}}^{(1)}(i), \overline{\bar{A}}^{(2)}(i, j)$ etc. indicates $\overline{\bar{A}}^{(1)}, \overline{\bar{A}}^{(2)}$ etc. are to be evaluated with the fields $\bar{v}_{i}, \bar{v}_{j}$ and so on. 
It is usual in perturbation calculations of this kind to require the first term in the velocity expansion to satisfy the boundary conditions exactly. The higher order terms must then vanish on the boundary; however, the boundary stresses associated with these fields do not vanish. Hence these terms will generate corrections to the forces and torques acting on the boundary. Such perturbation schemes will be called force (or torque) perturbations. In this work the inverse of force perturbations, called velocity perturbations, will be used throughout.

Let $S_{1}$ be the surface of a rigid body which moves with velocity $\bar{U}+\bar{\Omega} \times \bar{r}$ relative to an outer boundary or container whose surface is $S_{2}$. For the fluid contained in the region between $S_{1}$ and $S_{2}$ the boundary conditions for velocity perturbations are to be satisfied as follows

$$
\bar{v}_{i}=\bar{U}_{i}+\bar{\Omega}_{i} \times \bar{r} \text { on } S_{1}, \bar{v}_{i}=0 \text { on } S_{2} \text {. }
$$

The velocity perturbations $\bar{U}_{i}$ and $\bar{\Omega}_{i}$ on $S_{1}$ are determined by force and torque conditions given by

$$
\begin{aligned}
\bar{F}=- & \int_{S_{1}} \bar{t}_{1} \mathrm{dA}, \bar{L}=-\int_{S_{1}} \bar{r}_{1} \bar{t}_{1} \mathrm{dA} \\
& \int_{S_{1}} \bar{t}_{i} \mathrm{dA}=\int_{S_{1}} \bar{r}_{1} \bar{t}_{i} \mathrm{dA}=0,
\end{aligned}
$$

and for $i>1$

where $\bar{F}$ and $\bar{L}$ are respectively the force and torque exerted by the fluid on $S_{1}$. The stress vectors $\bar{t}_{i}$ are given by

$$
\bar{t}_{i}=-\bar{n}_{i}+\mu_{0} \bar{n}^{\prime} \overline{\bar{A}}^{(1)}(i)+\bar{n} \cdot \overline{\bar{i}}_{i}(i-1, i-2, \ldots, 1) \text {, }
$$


where $\bar{n}$ is the unit normal directed out of the fluid surface. Since the fields $\bar{V}_{1}, P_{1}$ satisfy the homogeneous stokes equation, $\bar{U}_{1}$ and $\bar{\Omega}_{1}$ are respectively the translational and rotational velocities with which the particle would move in a Newtonian fluid of viscosity $\mu_{0}$ when subjected to a force $-\bar{F}$ and a torque $-\bar{L}$.

When $S_{2}$ is infinitely far from $S_{1}$ the velocity contributions in (3.6) are denoted by $\bar{U}_{\infty j}, \bar{\Omega}_{\infty i}$. The sphere is the only case for which some of these terms are known. From the force and torque formulae of Giesekus [1963] it is possible to find by inversion ${ }^{2}$ :

$$
\begin{aligned}
& \bar{u}_{\infty_{1}}=-\frac{\bar{F}}{6 \pi \mu_{0}^{a}}, \quad \bar{u}_{\infty_{2}}=0 \text {, } \\
& \bar{U}_{\infty 3}=\frac{1}{175}\left[\frac{942}{13}\left(\frac{{ }^{\beta}{ }^{+\beta} 3}{\mu_{0}}\right)-\left(\frac{\alpha_{1}+\alpha_{2}}{\mu_{0}}\right)^{2}-\frac{258}{143} \frac{\beta_{1}}{\mu_{0}}\right]\left(\frac{F}{6 \pi \mu_{0} a^{2}}\right)^{2} \frac{\bar{F}}{6 \pi \mu_{0} a}, \bar{U}_{\infty 4}=0, \\
& \bar{U}_{5}=O\left(F^{5}\right) \text {. } \\
& \bar{\Omega}_{\infty 1}=-\frac{\bar{L}}{8 \pi \mu_{0} a^{3}}, \bar{\Omega}_{\infty 2}=0 \text {, } \\
& \bar{\Omega}_{\infty 3}=\frac{2}{15}\left[36\left(\frac{\beta_{2}+\beta_{3}}{\mu_{0}}\right)-\left(\frac{\alpha_{1}+\alpha_{2}}{\mu_{0}}\right)\right]\left(\frac{L}{8 \pi \mu_{0} a^{3}}\right)^{2} \frac{\bar{L}}{8 \pi \mu_{0} a^{3}}, \bar{\Omega}_{\infty 4}=0, \bar{\Omega}_{\infty 5}=0\left(L^{5}\right) \text {. }
\end{aligned}
$$

The above formulae are valid only for translation induced by a force alone or rotation induced by a torque alone. When both $\overline{\mathrm{F}} \neq 0, \overline{\mathrm{L}} \neq 0$ interaction terms appear in $\bar{U}_{\infty 3}$ and $\bar{\Omega}_{\infty 3^{\circ}}$. Giesekus has calculated these when $\bar{F}$ and $\bar{L}_{\infty}$ are

\footnotetext{
$2 \bar{U}_{\infty \ell 4}$ and $\bar{\Omega}_{\infty \text { l }}$ are not given, but they can be shown to vanish with the use of symmetry arguments.
} 
parallel, but his results appear to be in error (Caswell [1967]).

The calculation of wall effects depends crucially on the behavion of the fields $\bar{V}_{\infty i}, P_{\infty i}$ far from $S_{1}$. Brenner [1964a] has shown that the Newtonian fields have the asymptotic expansions

$$
\begin{aligned}
& \bar{v}_{\infty 1+}+\bar{V}_{f}+o\left(r^{-2}\right), \bar{v}_{f}=-\left(\overline{\bar{I}}+\frac{\bar{r} \bar{r}}{r^{2}}\right) \cdot \frac{\bar{F}}{8 \pi \mu_{o} r}, \\
& p_{\infty I}+p_{f}+o\left(r^{-3}\right), p_{f}=-\frac{\bar{r} \cdot \bar{F}}{4 \pi r^{3}},
\end{aligned}
$$

where the origin of the radius vector $\bar{r}$ is imbedded in the moving body $S_{1}$. The choice of an origin in the moving particle requires the corotational derivative (2.3) to be written with the velocity relative to that origin. Hence from $(2.2)$ and $(2.3) \overline{\bar{A}}^{(2)}$ can be expanded as

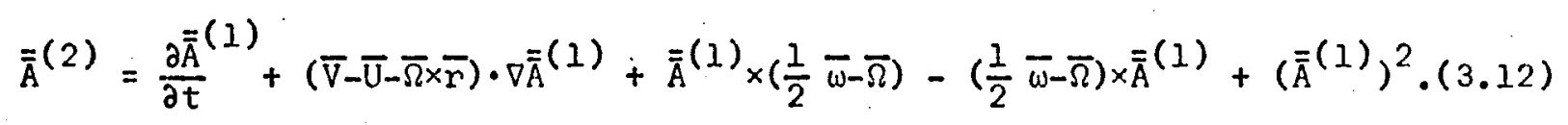
It follows from $(3.4),(3.5),(3.11 a)$ and $(3.12)$ that the $\overline{\bar{\tau}}_{\infty i}$ are $0\left(r^{-3}\right)$ as $r$ becomes large. It then also follows from the force condition ( $3.7 \mathrm{~b})$ and the divergence theorem that for $i>1 \bar{v}_{\infty i}, p_{\infty i}$ can be at most $0\left(r^{-2}, r^{-3}\right)$ as $r \rightarrow \infty$.

With the use of (3.11) and (3.12) it can be shown that the inhomogeneous stress tensors $\overline{\bar{\tau}}_{\infty i}$ may be written as

$$
\begin{aligned}
\overline{\bar{\tau}}_{\infty i}= & -\alpha_{1}\left\{\left(\bar{U}_{\infty i-1}+\bar{\Omega}_{\infty i-1} \times \bar{r}\right) \cdot \nabla \overline{\bar{A}}(1)(\infty 1)+\overline{\bar{A}}^{(1)}(\infty 1) \times \bar{\Omega}_{\infty i-1}-\bar{\Omega}_{\infty i-1} \times \overline{\bar{A}}^{(1)}(\infty 1)\right\} \\
& +\bar{\tau}_{\infty i}^{* *}
\end{aligned}
$$

where $\bar{\tau}_{\infty i}^{*}-O\left(r^{-4}\right)$ as $r+\infty$,

and $\overline{\bar{A}}^{(1)}(\infty 1)$ implies $\overline{\bar{A}}^{(1)}$ is to be evaluated with $\bar{V}_{\infty 1}$. Because of the form of (3.13) it is useful to construct new fields $\bar{v}_{f i}, p_{f i}(i>1)$ with 
$\bar{v}_{f i}=\frac{\alpha_{1}}{\mu_{0}}\left\{\left(\bar{u}_{i-1}+\bar{\Omega}_{i-1} \times \bar{r}\right) \cdot \nabla \bar{v}_{1}+\bar{v}_{1} \times \bar{\Omega}_{i-1}\right\}, p_{f i}=\frac{\alpha_{1}}{\mu_{0}}\left\{\left(\bar{u}_{i-1}+\bar{\Omega}_{i-1} \times \bar{r}\right) \cdot \nabla_{p_{1}}\right\}$.

These fields satisfy the homogeneous Stokes equation, and when $S_{2}$ is the sphere at infinity the corresponding stress fields $\overline{\mathrm{T}}_{\text {cofi }}$ approach the first term in (3.13) as $r$ becomes large. Furthermore, $\bar{V}_{\infty f i}, p_{\infty f i}$ do not contribute to the force or torque on $S_{1}$. It is the asymptotic properties of the $\tau_{\infty i}$ which permits the analysis for wall effects to be carried out without the need for complicated calculations.

4. The Green's Function Solution

The solution of the Stokes equation in terms of a generalized Green's function was originally worked out by Lorentz [Oseen 1927]. Recently, Williams [1966] has used this solution in his investigation of the effect of finite boundaries on the Stokes drag in Newtonian flow. Here these results are extended to include Nth order viscoelastic flow.

In an unbounded region the Green's "velocity" tensor and "pressure" vector are given respectively by (Oseen [1927])

$$
\begin{aligned}
& \overline{\bar{Q}}_{I}\left(\bar{r}, \bar{r}^{\prime}\right)=\overline{\bar{I}} \nabla^{2}\left|\bar{r}-\bar{r}^{\prime}\right|-\nabla \nabla\left|\bar{r}-\bar{r}^{\prime}\right|, \\
& \overline{\mathrm{P}}_{I}\left(\bar{r}, \bar{r}^{\prime}\right)=-\mu_{0} \nabla\left(\nabla^{2}\left|\bar{r}-\bar{r}^{\prime}\right|\right),
\end{aligned}
$$

where $\bar{r}$ and $\bar{r}^{\prime}$ are the radius vectors of two arbitrary points $X$ and $X^{\prime}$ respectively. The gradient symbols $\nabla$ and $\nabla^{\prime}$ denote differentiation with respect to the components of $\bar{r}$ and $\bar{r}^{\prime}$ respectively. The fields $\bar{Q}_{I}, \bar{P}_{I}$ satisfy equations of "motion" and "continuity" 


$$
\mu_{0} \nabla^{2} \bar{Q}_{I}-\nabla \bar{P}_{I}=-8 \pi \mu_{0} \overline{\bar{I}} \delta\left(\bar{r}-\bar{r}^{\prime}\right), \quad \nabla \cdot \overline{\bar{Q}}_{I}=0
$$

respectively. If the flow is confined to the region. between a particle $S_{1}$ and an outer surface $S_{2}$ then to $\overline{\bar{Q}}_{I}, \overline{\mathrm{P}}_{I}$ are added the fields $\overline{\bar{Q}}_{I I}\left(\bar{r}, \bar{r}^{\prime}\right)$, $\overline{\mathrm{P}}_{\text {II }}\left(\vec{r}, \bar{r}^{\prime}\right)$ which satisfy the homogeneous form of $(4.3)$ and the boundary condition

$$
\overline{\bar{Q}}=\overline{\bar{Q}}_{I}+\overline{\bar{Q}}_{I I}=0 \text { on } S_{2} \text {. }
$$

The fields $\overline{\bar{Q}}, \overline{\mathrm{P}}$ so defined are the Green's functions which deliver the solution of the Stokes equation (3.3) at points in the interior of $S_{2}$. As with the scalar Green's functions of potential theory $\overline{\bar{Q}}$ satisfies a reciprocity relation when $\vec{r}$ and $\vec{r}^{\prime}$ are interchanged (Viliat [1943])

$$
\bar{Q}\left(\bar{r}, \bar{r}^{\prime}\right)=\bar{Q}^{\dagger}\left(\bar{r}^{\prime}, \bar{r}\right) \text {. }
$$

where t denotes the transpose. By inspection $j$.t can be seen that $\overline{\bar{Q}}_{I}$ satisfies ( 4.5$)$, and hence the reciprocity relation is, in fact, a condition to be imposed on $\overline{\bar{Q}}_{I I}$. It is clear from (4.1), (4.2) and (4.4) that $\overline{\bar{Q}}_{I I}$ is determined solely by the nature of the surface $S_{2}$, and is regular everywhere within the region enclosed by $i t$, including the volume occupied by $S_{1}$.

If the inhomogeneous part of (3.3) is regarded as a body force then the velocity $\bar{v}_{i}$ at a field point $X^{\prime}$ can be written directly from Oseen's [1927] work as

$$
\begin{aligned}
8 \pi \mu_{0} \bar{v}_{i}\left(X^{\prime}\right) & =\int_{S_{1}+S_{2}}\left\{\left(-p_{i} \bar{n}+\mu_{0} \frac{\partial \bar{v}_{i}}{\partial n}\right) \cdot \bar{Q}-\bar{v}_{i} \cdot\left(-\bar{n} \bar{P}+\mu_{0} \frac{\partial \bar{Q}}{\partial n}\right)\right\} d A \\
& +\int_{v}\left(\nabla \cdot \bar{\tau}_{i}\right) \cdot \overline{\bar{Q}} d v,
\end{aligned}
$$

where $\bar{n}$ is the unit normal directed out of the fluid surface, and $x$ is now 
a variable point in the region between $S_{1}$ and $S_{2}$. With the identity

$$
\left(\nabla \cdot \overline{\bar{\tau}}_{i}\right) \cdot \overline{\bar{Q}}=\nabla \cdot\left(\overline{\bar{\tau}}_{i} \overline{\bar{Q}}\right)-\overline{\bar{\tau}}_{i}: \nabla \overline{\bar{Q}}, \cdot
$$

the divergence theorem, and the boundary conditions for $\bar{V}_{i}$ and $\overline{\bar{Q}}$ on $S_{2}$, the solution $(4.6)$ can be put into the form

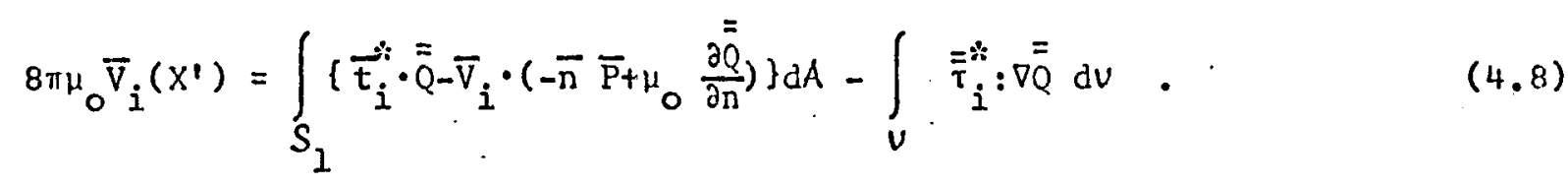
Here $\stackrel{-*}{\tau}_{i}^{*}$ and $\vec{t}_{i}^{*}$ are defined by

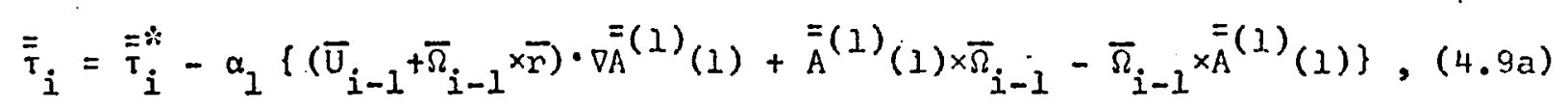

$\bar{t}_{i}=\ddot{t}_{i}-\bar{t}_{f i}$

where $\bar{t}_{f i}$ is the stress vector calculated from the fields $\bar{v}_{f i}, p_{f i}$ (3.15). Whenever $\bar{v}_{i}$ is a rigid motion on $S_{1}$ the second term in the surface integral can be shown to vanish with the use of the divergence theorem and the properties of $\overline{\bar{Q}}_{I}$ and $\overline{\bar{Q}}_{I I}$.

By arguments entirely analogous to those used to arrive at (4.8) it can be shown that the corresponding pressure field is given by ${ }^{3}$

$$
4 \pi p_{i}\left(X^{\prime}\right)=\int_{S_{1}} \nabla \cdot \phi \cdot \overrightarrow{t_{i}^{*}} \mathrm{dA}-\int_{v} \overline{\bar{\tau}}_{i}^{*}: \nabla \nabla \phi \mathrm{d} v+4 \pi \bar{\tau}_{i}^{*}: \overline{\bar{I}} / 3
$$

where $\phi\left(\bar{r}, \bar{r}^{\prime}\right)$ is the scalar Green's function for Laplace's equation given by

$$
\phi=\phi_{I}+\phi_{I I}, \phi_{I}=1 /|\bar{r}-\bar{r}|,
$$

$\overline{3}$ In (4.10) $\bar{v}$ has been taken as a rigid motion on $S_{1}$. 
and $\phi_{I I}$ is a regular solution of Laplace's equation which satisfies the boundary condition

$$
\nabla \phi_{I I}=-\nabla \phi_{I} \quad \text { on } \quad S_{2}
$$

As with $Q_{I I}, \phi_{I I}$ is determined solely by the properties of $S_{2}$. When $S_{2}$ recedes to infinity it is denoted by $S_{\infty}$, and $\overline{\bar{Q}}$ then reduces to $\bar{Q}_{I}$. If the field point $X^{\prime}$ is restricted to lie on $S_{I}$ then, because of the boundary conditions, $(4.8)$ reduces to the following set of integral equations which express the boundary value problem for the fields $\bar{v}_{\infty_{i}}, p_{\infty_{i}}$ :

$$
8 \pi \mu_{0}\left(\bar{U}_{\infty 1}+\bar{\Omega}_{\infty 1} \times \bar{r}\right)=\int_{S_{1}} \bar{t}_{\infty I} \cdot \bar{Q}_{I} \mathrm{dA},
$$

and for $i>1$

$$
8 \pi \mu_{0}\left(\bar{U}_{\infty i}+\bar{\Omega}_{\infty i} \times \bar{r}\right)=\int_{S_{1}} \bar{t}_{\infty i}^{*} \cdot \overline{\bar{Q}}_{I} \mathrm{dA}-\int_{v} \bar{\tau}_{\infty i}^{* *}: \nabla \overline{\bar{Q}}_{I} \mathrm{~d} v+0\left(l^{-3}\right),
$$

where $\ell$ is the characteristic dimension of $S_{2}$. For reasons soon to become apparent the volume integral in $(4.14)$ has been evaluated over the finite volume $v$ enclosed by $S_{2}$ rather than the whole exterior $v_{\infty}$. The error. introduced by the neglect of the integral over $v_{\infty}-v$ is estimated from the far field behavior of $\overline{\bar{\tau}}_{\infty i}^{*}$ as given by (3.24).

In the case of $S_{2}$ located at a finite distance from $S_{1}$ Williams [1966] expands $\overline{\bar{Q}}_{I I}$ in a Taylor's series about some origin $\circ$ in $S_{1}$.

$$
\overline{\bar{Q}}_{I I}=\overline{\bar{Q}}_{I I}^{\circ}+\bar{r} \cdot \nabla_{I I}^{=0}+\bar{r}^{\prime} \cdot \nabla \cdot \nabla_{I I}^{=0}+o\left(l^{-3}\right),
$$

where the superscipt ${ }^{\circ}$ implies the quantities are evaluated at the origin 0 . From the reciprocity relation $(4.5)$ it follows that $\overline{\mathrm{Q}}_{\mathrm{II}}$ is symmetric, and that $\nabla^{\prime}=0$ iI is equal to $\nabla_{I I}^{=0}$. It will be seen below that $\stackrel{0}{Q}_{I I}, \nabla_{I I}^{=0}$, etc. 
must be $0\left(l^{-1}, l^{-2}\right.$, etc. $)$ on dimensional grounds. Williams noted that $\nabla Q_{\text {II }}$ vanishes whenever the origin is located at a point through which may be drawn three perpendicular axes of symmetry of $S_{2}$. With the restriction to symmetric boundaries of this kind it is found from (4.15) and from the boundary and force conditions $(3.6)$ and $(3.7 a)$ that $(4.8)$ reduces to the set of integral equations:

$$
8 \pi \mu_{0}\left(\bar{U}_{1}+\bar{\Omega}_{1} \times \bar{r}\right)+\bar{F} \cdot \bar{Q}_{I I}^{0}=\int_{S_{1}} \bar{t}_{1} \cdot \overline{\bar{Q}}_{I} \mathrm{dA}+0\left(l^{-3}\right),
$$

and for $i>1$

$$
\begin{gathered}
8 \pi \mu_{0}\left(\bar{U}_{i}+\bar{\Omega}_{i} \times \bar{r}\right)-\bar{Q}_{I I}^{=0} \cdot \int_{S_{1}} \bar{t}_{f i} \mathrm{dA}=\int_{S_{I}} \bar{t}_{i}^{i} \cdot \overline{\bar{Q}}_{I} \mathrm{dA}-\int_{v} \overline{\bar{\tau}}_{i}: \nabla \overline{\bar{Q}_{I}} \mathrm{dV} \\
+0\left(e^{-3}\right),
\end{gathered}
$$

A comparison of $(4.1 .6)$ and $(4.17)$ with $(4.13)$ and $(4.14)$ shows that the fields $\bar{v}_{i}, p_{i}$ satisfy, to $O\left(l^{-3}\right)$, the same boundary value problem as the fields $\bar{v}_{\infty i}, P_{\infty i}$ provided

$$
\bar{U}_{\infty 1}=\bar{U}_{1}+\overline{\mathrm{F}} \cdot \overline{\mathrm{Q}}_{\mathrm{II}}^{=0} / 8 \pi \mu_{0}+0\left(e^{-3}\right), \bar{\Omega}_{\infty 1}=\bar{\Omega}_{1}+\dot{0}\left(\ell^{-3}\right),
$$

and for $i>1 \quad \bar{u}_{\infty i}=\bar{u}_{i}+0\left(e^{-3}\right), \bar{\Omega}_{\infty i}=\bar{\Omega}_{i}+0\left(e^{-3}\right)$.

The integral of $\bar{t}_{f i}$ over $S_{1}$ in (4.17) vanishes because to $o\left(l^{-3}\right) \bar{t}_{f i}$ is given by $\bar{t}_{\text {offi }}$ (see the remarks following $(3.15)$ ). The sum of the $\bar{U}_{i}, \bar{\Omega}_{i}$ and the $\bar{U}_{\infty i}, \bar{\Omega}_{\infty i}$ respectively yields (i.1), which relates the velocity $\bar{U}, \bar{\Omega}$ of $S_{1}$ in a bounded region with its velocity $\bar{U}_{\infty}, \bar{\Omega}_{\infty}$ in an unbounded region, the force and torque being identical in each case. In order to obtain ( $i .1$ ) it is necessary to note that the wall effect tensor $\overline{\bar{k}}$ is related to $\overline{\bar{Q}}_{I I}$ by

$$
\bar{k}=-3 \ell_{I I}^{=0} \text {. }
$$


The symmetry of $\overline{\bar{k}}$, which follows, directly from $(4.5)$ has previously been established by Brenner [1964a] using a different approach. Values of some of the components of $\overline{\bar{k}}$ for certain geometries are given in Happel and Brenner [1965].

\section{Asymmetric Configurations}

The configurations of $S_{1}$ and $S_{2}$ for which the terms $O\left(\ell^{-2}\right)$ in $(4.15)$ do not vanish will now be considered. The boundary value problem generated by substituting (4.15), including terms in $\nabla_{\mathrm{Q}}=0$, into (4.8) cannot be. satisfied to $0\left(l^{-3}\right)$ by the fields $\bar{V}_{\infty i}, P_{\infty i}$ alone. The integral equation for the Newtonian fields $\bar{v}_{1}, p_{1}$ are found to be

$$
8 \pi \mu_{0}\left\{\bar{U}_{1}-\delta \bar{U}_{1}+\left(\bar{\Omega}_{1}-\delta \bar{\Omega}_{1}\right) \times \bar{r}_{0}^{\prime}-\bar{r}_{0}^{\prime} \cdot \overline{\bar{s}}\right\}=\int_{S_{1}} \bar{t}_{1} \cdot \overline{\bar{Q}}_{I} \mathrm{dA}+0\left(l^{-3}\right),
$$

where

$$
\begin{aligned}
8 \pi \mu_{0} \delta \bar{U}_{I} & =-\bar{F} \cdot \overline{\bar{Q}}_{I I}^{0}-\frac{1}{2} \bar{L} \cdot \nabla \times \bar{Q}_{I I}+\frac{1}{2} \int_{S_{1}}\left(\bar{t}_{I} \bar{r}_{0}+\bar{r}_{0} \bar{t}_{I}\right): \nabla Q_{I I} \mathrm{dA}, \\
\delta \bar{\Omega}_{I} & =-\nabla \times \bar{Q}_{I I}^{=0} \cdot \bar{F} / I 6 \pi \mu_{0}, \\
\overline{\bar{s}} & =-\left(\nabla \overline{\bar{Q}}_{I I}^{0}+{ }_{\nabla Q_{I I}}^{=0}\right) \cdot \bar{F} / 26 \pi \mu_{0} .
\end{aligned}
$$

The solution of $(5.1)$ can be written as the superposition of $\bar{v}_{\infty I}, p_{\infty 1}$ and the perturbation field $\bar{V}_{\infty 1}^{\prime \prime}, p_{\infty 1}^{\prime \prime}$ required to satisfy motion in an unbounded shear field. These fields satisfy the boundary conditions:

$\bar{v}_{\infty 1}, \bar{V}_{\infty 1}^{\prime \prime}=\bar{U}_{\infty 1}+\bar{\Omega}_{\infty 1} \times \bar{r}, \bar{U}_{\infty 1}^{\prime \prime}+\bar{\Omega}_{\infty 1}^{\prime \prime} \times \bar{r} \quad$ respectively on $S_{1}$,

and $\bar{v}_{\infty 1}=0, \quad \bar{v}_{\infty 1}^{\prime \prime}=\bar{r} \cdot \bar{s}$ on $S_{\infty}$, 
where $\overline{\bar{S}}$ is a symmetric constant tensor such that $\overline{\bar{S}}: \overline{\bar{I}}=0$. The force and torque on $S_{1}$ are given by

$$
\bar{F}=-\int_{S_{1}} \bar{t}_{\infty 1} \mathrm{dA}, \overline{\mathrm{L}}=-\int_{S_{1}} \overline{r \times \bar{t}_{\infty 1}} \mathrm{dA},
$$

from which it follows that

$$
\vec{v}_{\infty 1}^{\prime \prime}+\bar{r} \cdot \overline{\bar{s}}+O\left(r^{-2}\right), p_{\infty 1}^{\prime \prime} \rightarrow O\left(r^{-3}\right) \text { as } r \rightarrow \infty
$$

From $(4.8),(5.3),(5.4)$, and $(5.6)$ the integral equations which express the boundary value problem for these fields are found from (4.8) to be

$$
\begin{aligned}
& 8 \pi \mu_{0}\left(\bar{U}_{\infty 1}+\bar{\Omega}_{\infty 1} \times \bar{r}_{0}^{\prime}\right)=\int_{S_{1}} \bar{t}_{\infty 1} \cdot \bar{Q}_{I} d A
\end{aligned}
$$

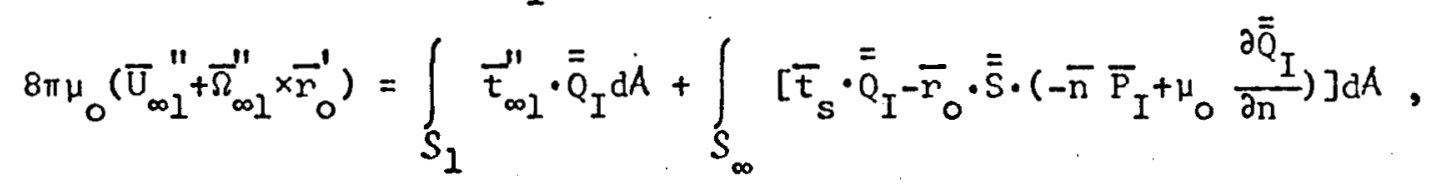

where $\overline{\mathrm{t}}_{s}=2 \mu_{0} \overline{\overline{\mathrm{S}}} \cdot \overline{\mathrm{n}}$. A uniform shear field $\dot{\bar{r}}_{0} \cdot \overline{\overline{\mathrm{S}}}$ is defined everywhere within $S_{\infty}$, and hence from (4.8) it can be formally expressed as

$$
8 \pi \mu_{0} \bar{r}_{0}^{\prime} \cdot \overline{\bar{s}}=\int_{S_{\infty}}\left[\bar{t}_{s} \cdot \overline{\bar{Q}}_{I}-\bar{r}_{0} \cdot \overline{\bar{s}} \cdot\left(-\bar{n} \bar{P}_{I}+\mu_{0} \frac{\partial \overline{\bar{Q}}}{\partial \mathrm{n}}\right)\right] \mathrm{d} A .
$$

From (5.10) it follows that to $0\left(l^{-3}\right)(5.1)$ is the superposition of (5.7) and (5.8) with

$$
\bar{U}_{1}-\delta \bar{U}_{1}=\bar{U}_{\infty 1}+\bar{U}_{\infty 1}^{\prime \prime}, \bar{\Omega}_{1}-\delta \bar{\Omega}_{1}=\bar{\Omega}_{\infty 1}+\bar{\Omega}_{\infty 1}^{\prime \prime} .
$$

Brenner [1.964c] has given a general analysis of the motion of particles in shear fields of Newtonian fluids, and from his results it can be shown that 


$$
\begin{aligned}
& \mu_{0} \bar{U}_{\infty 1}=-\left(\overline{\bar{K}-\overline{\bar{C}}}+\cdot \overline{\bar{R}}_{0}^{-1} \cdot \overline{\bar{C}}_{0}\right)^{-1} \cdot\left(\overline{\bar{F}}-\overline{\bar{C}}_{0}^{\dagger} \cdot \overline{\bar{R}}_{0}^{-1} \cdot \bar{L}\right) \text {, } \\
& \vec{U}_{\infty I}^{\prime \prime}=-\left(\overline{\bar{K}}-\overline{\bar{C}}_{0}^{\dagger} \cdot \overline{\bar{R}}_{0}^{-1} \cdot \overline{\bar{C}}\right)^{-1} \cdot\left(\overline{\bar{\Phi}}{ }_{0}-\overline{\bar{C}}+\cdot \overline{\bar{R}}_{0}^{-1} \cdot \overline{\bar{\sigma}}_{0}\right): \overline{\overline{\mathrm{S}}}, \\
& \mu_{0} \overline{\bar{R}}_{\infty 1}=-\left(\overline{\bar{R}}_{0}-\overline{\bar{C}}_{0} \cdot \overline{\bar{K}}^{-1} \cdot \overline{\bar{C}}_{0}^{\dagger}\right)^{-1} \cdot\left(\bar{L}-\overline{\bar{C}} \cdot \overline{\bar{K}}^{-1} \cdot \bar{F}\right), \\
& \bar{\Omega}_{\infty 1}^{\prime \prime}=-\left(\overline{\bar{R}}_{0}-\overline{\bar{C}}_{0} \cdot \overline{\bar{k}}^{-1} \cdot \overline{\bar{c}}_{0}^{\dagger}\right)^{-1} \cdot\left(\overline{\bar{\sigma}}_{0}-\overline{\bar{C}}_{0} \cdot \overline{\bar{K}}^{-1} \cdot \overline{\bar{\Phi}}_{0}\right): \overline{\bar{S}} \cdot
\end{aligned}
$$

The dyadics $\overline{\bar{K}}, \overline{\bar{C}}_{0}$ and $\overline{\bar{R}}_{0}$ are respectively the resistance, coupling and rotation dyadics which, for a Newtonian fluid, determine the force and torque on a rigid body undergoing translation and rotation in ar unbounded region. The shear force triadic $\overline{\bar{\phi}}_{0}$ and the shear torque triadic $\overline{\bar{\sigma}}_{0}$ determine the additional force and torque due to a shear field. The subscript o denotes polyadics which are origin dependent, and formulae for transforming these quantities to a new origin can be found in Brenner's papers $[1964 a, 1964 c]$.

To determine the velocity correction $\delta \bar{U}_{1}$ the integral in (5.2a) must be evaluated. From (5.9) and with the use of Brenner's [1964c] formalism it is easily shown that

$$
\frac{l}{2} \int_{S_{1}}\left(\bar{t}_{1} \bar{r}_{0}+\bar{r}_{0} \bar{t}_{1}\right) d A=\bar{U}_{1 \infty} \cdot \bar{\Phi}_{0}+\bar{\Omega}_{1 \infty} \cdot \bar{\sigma}_{0}+0\left(l^{-2}\right) .
$$

Since $\nabla \overline{\bar{Q}}_{\mathrm{II}}^{0}$ is $O\left(\ell^{-2}\right)(5.16)$ is sufficiently accurate for use in (5.1).

Because (5.1) involves only the Newtonian term it can be compared to some known results. Previous analyses by Brenner $[1962,1964 a]$ and by Williams [1966] have dealt with freely falling $(\bar{L}=0)$ particles. Neither of these authors has obtained explicit expressions for the terms of $o\left(l^{-2}\right)$ 
but rather have attempted to find symmetry criteria for the validity of the error estimate of (4.16). When the origin is the center of mass of a freely falling particle the following equation is obtained from (5.1), (5.2), and $(5.1 .2)$ to $(5.16)$

$\overline{\mathrm{U}}_{1}=\overline{\mathrm{U}}_{\infty 1}+\left(\overline{\mathrm{F}} \cdot \overline{\mathrm{k}} \ell+\overline{\mathrm{B}}^{\circ} \times \overline{\mathrm{F}}\right) / 6 \pi \mu_{0} \ell^{2}+0\left(\ell^{-3}\right)$

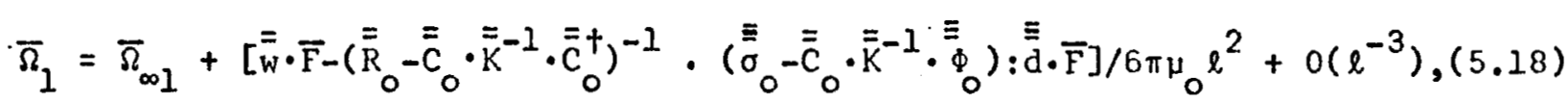
where $\overline{\mathrm{B}}^{\circ}=\bar{\varepsilon}: \overline{\bar{A}}{ }^{\circ}, \overline{\bar{\varepsilon}}$ is the alternating tensor, and

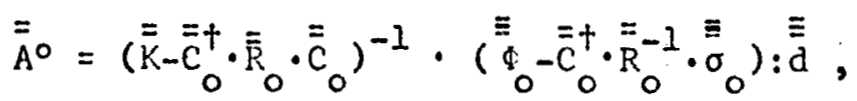

$$
\begin{aligned}
& \overline{\bar{w}}=-\frac{3}{8} \ell^{2} \nabla \times \overline{\bar{Q}}_{I I}^{\circ}, \quad \overline{\overline{\mathrm{d}}}=-\frac{3}{8} \ell^{2}\left(\nabla \overline{Q_{I I}}=0,+{ }_{\nabla Q_{I I}}^{=0}\right) .
\end{aligned}
$$

It should be noted that $\vec{A}^{\circ}$, and hence $\widehat{B}^{\circ}$, depends on the geometries of both $S_{1}$ and $S_{2}$ while the second order wall-effect tensors $\bar{w}$ and $\overline{\bar{d}}$ depend only on the shape of $S_{2}$. If the container is taken to have any arbitrary shape then the condition on the particle geometry for (5.19a) to vanish is

$$
\Phi_{0}-\overline{\bar{C}}+\frac{\overline{R_{0}}}{-1} \cdot \overline{\bar{\sigma}}_{0}=0 \text {, }
$$

where the origin dependent poladics are referred to the center of mass. From the transformation formulae of Brenner [1964c] it can be shown that in general it is not possible to find an origin for which (5.20) will always hold. Hence even with the adjustment of the mass distribution $\overline{\mathrm{B}}^{0}$ cannot in general be made to vanish. For highly symmetric bodies (5.20) may be satisfied with the origin at the center of symmetry. In particular, when $S_{1}$ has three mutually orthogonal symmetry planes $\bar{\Phi}_{0}=\overline{\bar{c}}_{0}=0$ (Brenner [1964c.]), and hence 
(5.17) reduces to (4.18) which is in accord with Williams [1966]. Another class of bodies for which $(5.20)$ is satisfied is that possessing helicoidal isotropy in which case $\overline{\bar{\Phi}}_{0}=\overline{\bar{\sigma}}_{0}=0$.

In his first paper on wall effects Brenner [1962] derived the onedimensional form of (4.18). He asserted that the error is $0\left(l^{-3}\right)$ provided the particle possesses three orthogonal symmetry planes, and moves with one of its principle axes parallel to a principle axis of the outer boundary. Later (Happel and Brenner [1965], p.291) the restrictions on the particle geometry were removed, and it was asserted that an origin always exists for which the error is $O\left(e^{-3}\right)$. This assertion is at odds with (5.17) as will be seen below where the case of an axisymmetric particle moving near a plane wall will be considered. Part of the difficulty of Brenner's assertion is that he does not define precisely the concept of a principle axis of the outer boundary. For the motion of a sphere a principle axis of $S_{2}$ could be defined as one for which $\bar{w} \cdot \bar{F}$ vanishes; however, this definition will not work for. particles with a lower degree of symmetry. Even if the term $0\left(\ell^{-2}\right)$ vanishes in $\bar{U}_{l}$, the corresponding term in $\bar{\Omega}_{1}$ will in general not vanish. The motion of a sphere near a plane wall is the notable exception to this rule.

For the non-Newtonian fields $\bar{v}_{i}, p_{i}, i>1$, it follows from $(3.6)$, (3.7) and (4.8) that to $O\left(l^{-3}\right)$ the boundary value problem will take the form

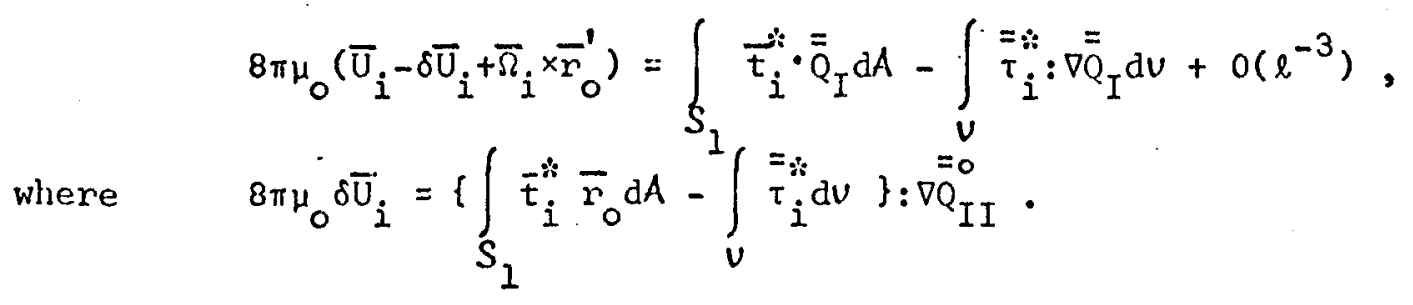

The solution of $(5.21)$ can be expressed, as in the case $i=1$, as the superposition of $\bar{v}_{\infty i}, p_{\infty i}$ and the perburbation fields $\bar{v}_{\infty i}^{\prime \prime}, p_{\infty i}$, which account for 
motion in a shear field. Since $\overline{\vec{S}}$ as given by $(5.2 c)$ is $O\left(l^{-2}\right)$ only the terms linear in this quantity are required for the solution of (5.21). The boundary conditions satisfied by these fields are rigid motion on $S_{1}$ as in $(5.3)$, and

$$
\bar{v}_{\infty i}=\bar{v}_{\infty i}^{\prime \prime}=0 \text { on } S_{\infty}
$$

Because the force and torque are given by $\bar{V}_{\infty 1}, p_{\infty l}$ the higher order fields must also satisfy

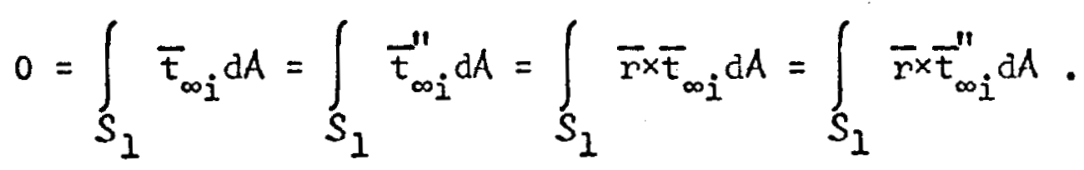

These force and torque conditions determine $\bar{U}_{\infty i}, \bar{U}_{\infty i}^{\prime \prime}, \bar{\Omega}_{\infty i}$ and $\bar{\Omega}_{\infty i}^{\prime \prime}$.

The only case for which any of these fields are known is the sphere. The author ${ }^{4}$ has calculated these fields for $i=2$ when the sphere is subjected simultaneously to a force and torque in any orientation. The velocity contributions are found to be:

$$
\begin{aligned}
& \bar{U}_{\infty 2}=0, \bar{U}_{\infty 2}^{\prime \prime}=-\left(\frac{\alpha_{1}+\alpha_{2}}{\mu_{0}}\right) \frac{\bar{F} \cdot \overline{\bar{S}}}{6 \pi \mu_{0} a}, \\
& \bar{\Omega}_{\infty 2}=0, \quad \bar{\Omega}_{\infty 2}^{\prime \prime}=-\left(\frac{\alpha_{1}+\alpha_{2}}{\mu_{0}}\right) \frac{\bar{L} \cdot \overline{\bar{S}}}{8 \pi \mu_{0} a^{3}} .
\end{aligned}
$$

With the use of symmetry arguments it can be shown that $\bar{U}_{\infty 3}^{\prime \prime}, \bar{\Omega}_{\infty 3}^{\prime \prime}$ have no terms linear in $\bar{S}$. Hence for the sphere $\bar{v}_{2 \infty}+\vec{V}_{2 \infty}^{\prime \prime}$ and $\bar{v}_{3 \infty}+\bar{V}_{3 \infty}^{\prime \prime}$ satisfy $(5.21)$ to $0\left(l^{-3}\right)$ provided

4 These calculations will be published in a forthcoming paper on particle motion in shear fields. 


$$
\begin{aligned}
& \bar{U}_{2}-\delta \bar{U}_{2}=-\left(\frac{\alpha_{1}+\alpha_{2}}{\mu_{0}}\right) \frac{\bar{F} \cdot \overline{\bar{S}}}{6 \pi \mu_{0}^{a}}, \bar{\Omega}_{2}=-\left(\frac{\alpha_{1}+\alpha_{2}}{\mu_{0}}\right) \frac{\bar{L} \cdot \overline{\bar{S}}}{8 \pi \mu_{0} a^{3}}, \\
& \bar{U}_{3}-\delta \bar{U}_{3}=\bar{U}_{3 \infty 0}, \quad \bar{\Omega}_{3}=\bar{\Omega}_{3 \infty} .
\end{aligned}
$$

The evaluation of the integral $(5.22)$ is greatly simplified with the use of a generalization of a reciprocal theorem of Lorentz (Oseen [1927]). For any two solutions $\bar{v}_{i}, p_{i}$ and $\bar{v}_{j}, p_{j}$ of the inhomogeneous stokes equations (3.3) the divergence theorem yields the following reciprocal relation

$$
\int_{V}\left\{\bar{\tau}_{j}: \nabla \bar{v}_{i}-\bar{\tau}_{i}: \nabla \bar{v}_{j}\right\} d V=\int_{S}\left\{\bar{t}_{j} \cdot \bar{v}_{i}-\bar{t}_{i} \cdot \bar{v}_{j}\right\} d A,
$$

where $S$ is the entire boundary of the volume $V$. When $S$ consists of $S_{1}$ and $S_{2}$, and $\bar{v}_{1}=\bar{r}_{0} \cdot \nabla \bar{Q}_{I I}^{0} \cdot \bar{c}$, where $\bar{c}$ is an arbitrary constant vector, the reciprocal theorem together with $(4.9)$ give $^{5}$

$$
\int_{V}^{=* *} \tau_{j}^{*} \mathrm{~d} V-\int_{S_{I}} \bar{t}_{j}^{*} \bar{r}_{0} \mathrm{dA}: \nabla Q_{I I}^{=0}=\int_{S_{\omega}}\left\{\bar{t}_{j}^{*} \bar{r}_{0}: \nabla Q_{I I}=0, \mu_{0} \bar{v}_{j} \bar{n}:\left(\nabla Q_{I I}+{ }^{+} \nabla Q_{I I}^{=0}\right)\right\} d A,
$$

where the error involved in the replacement of $S_{2}$ by $S_{\infty}$ is $O\left(l^{-3}\right)$.

The integral $(5.30)$ can be evaluated to $G\left(l^{-3}\right)$ with the $\bar{v}_{\infty j}, P_{\infty j}$ fields alone. For the sphere it can be shown from Giesekus [.1963] that $\overline{\mathrm{v}}_{\infty 2}, \mathrm{p}_{\infty 2}$ have the asymptotic expansions.

$$
\bar{V}_{2 \infty}=\frac{\left(\alpha_{1}+\alpha_{2}\right)}{12 \pi \mu_{0}^{2} a^{3}}\left\{\frac{\left(a^{2} \overline{F F} \cdot \nabla-\bar{L} \bar{L} \cdot \nabla\right)}{8 \pi \mu_{0}} \cdot\left(\frac{\overline{1}}{r}+\frac{\bar{r} r}{r^{3}}\right)\right\}+0\left(r^{-3}\right),
$$

\footnotetext{
5 The notation used above makes use of the clustering convention whereby $(\bar{a} \bar{b}):(\bar{c} \bar{d})=(\bar{a} \cdot \bar{d})(\bar{b} \cdot \bar{c})$. Also ${ }^{+} \bar{\nabla}_{I I}$ denotes the pre-transpose in which the
} .first two indices are interchanged. 


$$
\mathrm{P}_{2}=\frac{\left(\alpha_{1}+\alpha_{2}\right)}{12 \pi \mu_{0}^{2} a^{3}}\left\{\frac{\left(a^{2} \overline{F F} \cdot \nabla-\bar{L} \bar{L} \cdot \nabla\right)}{4 \pi} \cdot \frac{\bar{r}}{r^{3}}\right\}+o\left(r^{-4}\right)
$$

From these expansions the necessary integrals for use in (5.30) are calculated as

$$
\begin{aligned}
& \int_{S_{\infty}} \vec{t}_{\infty 2} \bar{r} d A=\frac{\left(\alpha_{1}+\alpha_{2}\right)}{60 \pi \mu_{0}^{2} a^{3}}\left[a^{2}\left(3 \overline{F F}-F^{2} \overline{1}\right)-\left(3 \bar{L}-L^{2} \overline{\bar{l}}\right)\right], \\
& \mu_{0} \int_{S_{\infty}} \bar{v}_{\infty 2} \bar{n} d A=-\frac{\left(\alpha_{1}+\alpha_{2}\right)}{180 \pi \mu_{0}^{2} a^{3}}\left[a^{2}\left(3 \bar{F} \bar{F}-F^{2} \bar{l}\right)-\left(3 \bar{L} \bar{L}-L^{2} \bar{l}\right)\right] .
\end{aligned}
$$

The fields $\bar{V}_{\infty 3}, P_{\infty 3}$ are $O\left(r^{-3}, r^{-4}\right)$, and so their integrals equivalent to (5.33) and (5.34) vanish. Higher order $\bar{\nabla}_{\infty j}, p_{\infty i}$ are not available.

For the sphere all the resistance polyadics in (5.12) to (5.14) are zero except $\overline{\bar{K}}$ and $\overline{\mathrm{R}}_{0}$ which are $6 \pi a \bar{l}$ and $8 \pi \mathrm{a} \overline{\bar{I}}$ respectively. From $(5.2)$, $(5.11),(5.19 b),(5.21),(5.27)$ and $(5.28)$ the velocity contributions $\bar{U}_{i}, \bar{\Omega}_{i}$ can be added to give

$$
\begin{array}{r}
\bar{U}=\bar{U}_{\infty}-\frac{a}{\ell} \bar{U}_{\infty 1} \cdot \bar{k}-\frac{4}{3} \frac{a^{3}}{\ell^{2}} \cdot \bar{w}-\frac{a}{l^{2}}\left(\frac{\alpha_{1}+\alpha}{\mu_{0}}\right)\left[\bar{U}_{\infty 1} \cdot \bar{d} \cdot \bar{U}_{\infty 1}+\left(\frac{8}{9} a^{2} \bar{\Omega}_{\infty 1} \bar{\Omega}_{\infty 1}-\frac{I_{\bar{U}}}{2} \bar{U}_{\infty 1}\right): \vdots \bar{d}\right]+ \\
0\left(l^{-3}, l^{-2} F^{4}, l^{-2} L^{4}, \ldots\right),
\end{array}
$$

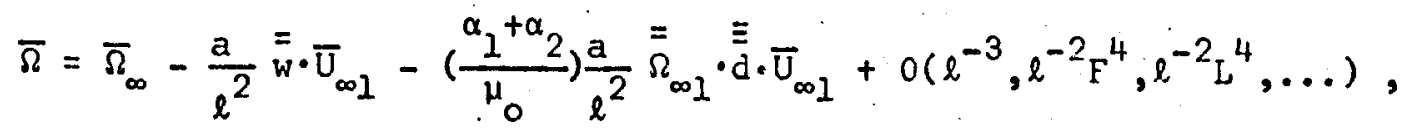

where $\bar{U}_{\infty 1}, \bar{\Omega}_{\infty 1}$, given by $(3.9)$ and $(3.10)$, are the velocities the sphere would attain in an unbounded Newtonian fluid of viscosity $\mu_{0}$ under the influence of the force $\bar{F}$ and torque $\bar{L}$. The error estimates in $(5.35)$ and (5.36) contain terms in $\ell^{-2}$ since for $i>3$ the non-Newtonian fields are not available. However since these terms are modified by fourth order products of $F$ and $L$ these equations are valid in the limit of small forces and torques. 
In experiments with freely falling particles this requirement is easily met by the use of small spheres which is also necessary to satisfy the condition for inertialess flow. Thus these formulae should be useful in falling sphere viscometry.

6. On the Quasi-Steady Approximation

In the preceding analysis all motions have been assumed to be steady. Obviously when a body moves in the vicinity of a wall the fluid motion cannot be steady in the strict sense except in centain special cases. For Newtonian fluids it can be shown with dimensional arguments that stokes equation remains valid provided the local and convected accelerations remain finite in the zero Reynolds number limit. In slow viscoelastic flow it is necessary to examine, in addition to the local acceleration, the partial time derivatives in the constitutive equation. Because of such terms the concept of quasisteady flow appears to be more restrictive than in the Newtonian case. Hence the analysis is restricted to the situation described below.

It is supposed that in an unbounded medium the body $S_{1}$ can undergo strictly steady motion under the influence of a constant force $\bar{F}$ and torque L. 6 This steady motion is disturbed by the approach of the body to a wall $S_{2}$ at a distance $\ell$. The speed of approach is $i$, and hence a characteristic approached time is $\ell / \dot{l}$. The dimensional analysis referred to above then shows the local acceleration to be $0\left(\frac{a}{l} R e\right)$, where $a$ is the particle dimension upon which $R e$ is based. Since the wall effect analysis requires $(a / \ell) \ll 1$, it is clear the local acceleration can be safely neglected in the zero Reynolds

6 For bodies of complex geometry such motions may not exist (cf. Brenner [2964b]). 
number limit. Therefore, the governing equations remain the inhomogeneous Stokes equations (3.3). However, the inhomogeneous stress tensors $\bar{\tau}_{i}^{\prime}$ now differ from their steady state values by certain partial time derivative terms. For instance, $=_{2}^{=1}$ includes the term $a_{1} \partial{ }_{A}^{(1)}(1) / a t$; thus the governing equation for the time dependent fields $\bar{v}_{2}^{\prime}, p_{2}^{\prime}$ is

$$
0=-\nabla p_{2}^{\prime}+\mu_{0} \nabla^{2} \bar{v}_{2}^{\prime}+\alpha_{1} \frac{\partial \nabla^{2} \bar{v}_{1}^{\prime}}{\partial t}+\nabla \cdot \tau_{2},
$$

where $\bar{\tau}_{2}$ is that part of $(3.4)$ which does not contain partial time derivatives. Since $\bar{V}_{1}^{\prime}$ satisfies the homogeneous Stokes equation it varies with time only through the velocity boundary values on $S_{1}$. A solution to (6.1) which satisfies the force and torque condition (3.7) is given by

$$
\bar{v}_{1}^{\prime}=\bar{v}_{1}, \bar{v}_{2}^{\prime}=\bar{v}_{2}-\frac{\alpha_{1}}{\mu_{0}} \frac{\partial \bar{v}_{1}^{\prime}}{\partial t}, \quad p_{2}^{\prime}=p_{2},
$$

where the unprimed fields satisfy the steady flow equations. on $S_{1} \bar{v}_{2}^{\prime}$ is given by

$$
\overline{\mathrm{V}}_{2}^{\prime}=\overline{\mathrm{U}}_{2}^{\prime}=\overline{\mathrm{U}}_{2}-\frac{\alpha_{1}}{\mu_{0}} \dot{\overline{\mathrm{U}}}_{1}
$$

The acceleration $\dot{\bar{U}}_{1}$ is obtained by time differentiation of (4.18) which results in 7

7 The force of the fluid on the particle $(3.7 \mathrm{a})$ has been taken as a constant in balance with the applied external force, such as that due to gravity. In an unsteady motion this cannot hold exactly. An estimate of the particle acceleration is furnished by $(6.4)$, and from the particle equation of motion it can be shown that the ratio (mass)(acceleration)/(fluid drag force) is $O\left(\operatorname{Re} \frac{\rho_{s}}{\rho} \frac{a^{2}}{l^{2}}\right)$. Here $\rho_{s}$ is the mass density of the solid. A similar argument can be used to justify the torque balance. 


$$
\dot{\bar{U}}_{1}=-\frac{\overline{\mathrm{F}} \cdot \overline{\mathrm{k}}}{6 \pi \mu_{0}} \frac{\dot{\imath}}{\ell^{2}} \text {. }
$$

Thus whenever $\dot{z}$ is $o(U) \bar{U}_{2}$ must be replaced by $\bar{U}_{2}^{\prime}$. The partial time derivatives in the $\bar{\tau}_{i}$ for $i>2$ contribute to the $\bar{U}_{i}$ only terms $0\left(l^{-3}\right)$, and to the same order all the $\bar{\Omega}_{i}$ are unaffected. Hence when all the contributions $\bar{U}_{i}^{\prime}, \bar{\Omega}_{i}^{\prime}$ are added the actual velocities $\bar{U}^{\prime}, \bar{\Omega}^{\prime}$ can be expressed as

$$
\bar{U}^{\prime}=\bar{U}+\frac{\alpha_{1}}{\mu_{0}} \frac{\bar{F} \cdot \bar{k}}{6 \pi \mu_{0}} \frac{i}{\ell^{2}}+0\left(\ell^{-3}\right), \bar{\Omega}^{\prime}=\bar{\Omega}+0\left(\ell^{-3}\right),
$$

where $\bar{U}, \bar{\Omega}$ are the velocities calculated from the steady. state equations which for the sphere are summarized by $(5.35)$ and $(5.36)$. When the particle arrives near the wall the above analysis may become invalid, not only because of the neglected terms $O\left(l^{-3}\right)$ in $(6.5)$, but more importantly because the stress will not necessarily be accurately described by the Rivlin-Ericksen theory. The velocity correction in $(6.5)$ should be regarded as a zeroth order account of stress relaxation.

\section{Examples of Wall Effects}

\section{a. The Plane .}

The volume $v$ is the half space on one side of the plane whose unit normal vector, $\bar{i}$, is directed into the fluid. For this case $\overline{\bar{Q}}_{\text {II }}$ is known exactly, and ran be written as (Oseen [1927] p. 114)

$$
\begin{aligned}
\overline{\bar{Q}}_{I I}\left(\bar{r}, \bar{r}^{\prime}\right) & =\nabla \nabla R-\overline{\bar{i}} \nabla^{2} R-2 \overline{r^{\prime}} \cdot \bar{i} \bar{i} \nabla(I / R)+\nabla(1 / R) \bar{i}-2 \bar{i} \bar{i} \bar{i} \cdot \nabla(1 / R) \\
& -\bar{r} \cdot \bar{i}(\nabla \nabla(i / R)-2 \bar{i} \cdot \nabla \nabla(\bar{l} / R) \bar{i})\},
\end{aligned}
$$

where $R=\left|\bar{r}-\bar{r}^{\prime}+2 \bar{i} \bar{r} \cdot \bar{i}\right|$, 
and the origin of $\bar{r}$ and $\overline{r^{\prime}}$ lies in the plane. The origin for the Taylor's expansion (4.15) lies at a distance $\ell$ from the plane, and from (7.1) this series gives

$\overrightarrow{\bar{Q}}_{I I}^{0}=-\frac{3}{4 \ell}[\overline{\bar{l}}+\bar{i} \bar{i}], \quad \nabla_{I I}^{=0}=\frac{3}{8 \ell^{2}}\left[\bar{i} \bar{i} \bar{i}-\overline{\bar{l}} \bar{i}+\overline{\bar{i}} \overline{\bar{l}}+(\overline{\bar{l}} \bar{i})^{\dagger}\right]$.

Hence the wall-effect tensors $(4.20)$ and (5.37) are

$$
\overline{\bar{k}}=\frac{9}{16}[\overline{\bar{l}}+\overline{\bar{i}} \bar{i}], \bar{w}=0, \overline{\bar{d}}=-\frac{9}{32}[\overline{\bar{i}} \bar{i} \bar{i}-\overline{\bar{l}} \bar{i}+\overline{\bar{i}} \overline{\bar{l}}+(\overline{\bar{i}} \bar{i})] .
$$

When terms of $O\left(l^{-3}\right)$ are neglected the appropriate value of $\dot{l}$ in. (6.5) is $\bar{U}_{\infty} \cdot \bar{i}$. When the above values for $\overline{\bar{k}}$ and $\overline{\bar{d}}$ are substituted in (5.35) and (5.36) the instantaneous velocity of a sphere can be expressed as

$$
\begin{aligned}
& \bar{U}^{\prime}=\bar{U}_{\infty}-\frac{9 a}{16} U_{\infty 1}\left(1+\frac{\alpha_{1}}{\mu_{0} l} \bar{U}_{\infty} \cdot \bar{i}\right)[\bar{g}+(\bar{g} \cdot \bar{i}) \bar{i}]+\frac{9}{64} \frac{a}{\ell^{2}}\left(\frac{\alpha_{1}+\alpha_{2}}{\mu_{0}}\right) U_{\infty 1}^{2}\left[\bar{i}(\bar{g} \cdot \bar{i})^{2}+3 \bar{i}-2 \bar{g} \cdot \bar{i}\right] \\
& +\frac{1}{4} \frac{a^{3}}{\ell^{2}}\left(\frac{\alpha_{1}+\alpha_{2}}{\mu_{0}}\right) \Omega_{\infty 1}^{2}\left[\bar{i}(\bar{h} \cdot \bar{i})^{2}-\bar{i}+2 \bar{h} \cdot \bar{i} \bar{h}\right]+0\left(\ell^{-3}, \ell^{-2} F^{4}, \ell^{-2} L^{4}, \ldots\right), \\
& \bar{\Omega}^{\prime}=\bar{\Omega}_{\infty}+\frac{9 a}{32 \ell^{2}}\left(\frac{\alpha_{1}+\alpha_{2}}{\mu_{0}}\right) U_{\infty 1} \Omega_{\infty 1}[\bar{i}(\bar{h} \cdot \bar{i} \bar{g} \cdot \bar{i}-\bar{h} \cdot \bar{g})+\bar{h} \cdot \overline{i g}-\bar{g} \cdot \bar{i} \bar{h}] \\
& \quad+0\left(\ell^{-3}, \ell^{-2} F^{4}, \ell^{-2} L^{4}, \ldots\right),(7.6)
\end{aligned}
$$

where $\bar{g}$ and $\bar{h}$ are unit vectors in the direction of $\bar{U}_{\infty I}$ and $\bar{\Omega}_{\infty I}$ (i.e. $-\bar{F}$ and -I) respectively. Several special cases of (7.6) best illustrate the nature of the non-Newtonian terms of $O\left(l^{-2}\right)$ :

i) Free fali with the force parallel to the plane

$$
\bar{u}_{11}=\bar{g}\left[U_{\infty}(F)-\frac{9}{16} \frac{a}{\ell} U_{\infty 1}\right], \bar{U}_{1}=\bar{i} \frac{27}{64} \frac{a}{\ell^{2}}\left(\frac{\alpha_{1}+\alpha_{2}}{\mu_{0}}\right) U_{\infty 1}^{2}, \bar{\Omega}=0 ;
$$


ii) Free fall with the force normal to the plane

$$
\bar{u}_{11}=0, \bar{u}_{1}=\bar{g}\left[U_{\infty}(F)-\frac{9}{8} \frac{a}{\ell}\left(1 \pm \frac{\alpha_{1}}{\mu_{0} \ell} U_{\infty}(F)\right) \pm \frac{9}{32} \frac{a}{\ell^{2}}\left(\frac{\alpha_{1}+\alpha_{2}}{\mu_{0}}\right) U_{\infty 1}^{2}\right], \bar{\Omega}=0,
$$

where $\bar{U}_{11}$ and $\bar{U}_{\perp}$ are respectively the components of velocity parallel and perpendicular to the plane. In $(7.8)$ the upper signs hold when the force is directed away from the wall and the lower signs when it is directed towards it.

iii) Rotation about a stationary axis parallel to the plane.

$$
\bar{\Omega}^{\prime}=\bar{\Omega}_{\infty}(L), \bar{U}^{\prime}=0, \bar{F}=-\frac{3}{2} \pi \frac{a^{4}}{l^{2}}\left(\alpha_{1}+\alpha_{2}\right) \Omega_{\infty 1}^{2} \bar{i} .
$$

iv) Rotation about a stationary axis perpendicular to the plane.

$$
\bar{\Omega}^{\prime}=\bar{\Omega}_{\infty}(L), \bar{U}^{\prime}=0, \bar{F}=3 \pi \frac{a^{4}}{\ell^{2}}\left(\alpha_{1}+\alpha_{2}\right) \Omega_{\infty 1}^{2} \bar{i} .
$$

In (7.9) and (7.10) the sphere is constrained not to translate. The force $\bar{F}$, calculated from (7.5) with: $\bar{U}^{\prime}=0$, is that exerted by the fluid on the sphere, and hence a force $-\bar{F}$ must be supplied to maintain the constraint. The normal stress coefficient $\alpha_{1}$ is negative (Truesdell and Noll [1965]) on theoretical grounds, and for polymer solutions $\alpha_{1}+\alpha_{2}$ is found to be positive. Hence when the force and torque vectors are parallel to the plane the wall effect produced by the normal stresses is to propel a freely translating sphere (7.7) away from the wall and to draw towards it a rotating sphere (7.9). When the force and torque vectors are normal to the plane the wall effects are more complicated. The normal stress terms tend to increase or decrease the velocity $(7.8)$ of a freely translating sphere depending on whether it leaves or approaches the wall. For the rotating sphere the effect is to push the sphere away from the wall. 
While (5.35) and (5.36) are valid only for spheres, (5.17) and (5.18) are valid for any geometry provided the fluid is taken to be Newtonian. As a specific example the free fall of an axisymmetric body in the vicinity of a plane wall will be studied. The necessary resistance polyadics are given in Brenner's papers as:

$$
\begin{aligned}
& \overline{\bar{k}}=k_{1} \bar{j} \bar{j}+k_{2}(\overline{\bar{l}}-\bar{j} \bar{j}), \quad R_{c}=R_{1} \overline{j j}+R_{2}(\bar{l}-\bar{j} \bar{j}), \\
& \equiv_{\Phi_{c}}=\bar{j} \overline{j j} \Phi_{1}+\left[\overline{\bar{l}} \bar{j}+(\overline{\bar{l}} \bar{j})^{\dagger}\right] \Phi_{2}, \quad \bar{\sigma}_{c}=\left[\overline{\bar{l}} \times \bar{j} \bar{j}+(\bar{l} \times \overline{j j})^{\dagger}\right] \sigma,
\end{aligned}
$$

where $\bar{j}$ is a unit vector parallel to the symmetry axis, and the subscript $c$ indicates the origin dependent polyadics are referred to the center of hydrodynamic stress where they take on their simplest form. In free fall the origin in (5.18) and (5.19) is of necessity the center of mass which is assumed to lie on the axis of symmetry. With the transformation formulae of Brenner it can be shown that the quantities $\overline{\mathrm{R}}_{0}-\overline{\overline{\mathrm{C}}} \cdot \overline{\overline{\mathrm{K}}}^{-1} \cdot \overline{\overline{\mathrm{C}}}_{0}^{+}, \bar{\sigma}_{0}-\overline{\overline{\mathrm{C}}} \cdot \overline{\overline{\mathrm{K}}}^{-1} \equiv \Phi_{0}$, and $\overline{\mathrm{L}}-\overline{\bar{C}}_{0} \cdot \overline{\bar{K}}^{-1} \cdot \overline{\mathrm{F}}$ are not origin dependent. This also follows directly from (5.14) and (5.15) since these quantities specify the angular velocity. From (5.17), (5.18), (5.19), (7.3) and Brenner's transformation formulae the velocity of an axisymmetric body near a plane wall in a Newtonian fluid is calculated as $\overline{\mathrm{v}}_{1}=\overline{\mathrm{U}}_{\infty 1}+\frac{3}{32 \pi \mu_{0} l}[\overline{\mathrm{F}}+\overline{\mathrm{i}} \cdot \overline{\mathrm{i}}]+$ $\left.\frac{3}{64 \pi \mu_{0} \ell^{2}}[\bar{i} \bar{F} \cdot \bar{j}-\bar{j} \bar{F} \cdot \bar{i}\}\left(1-(\bar{j} \cdot \bar{i})^{2}\right)\left(\frac{\Phi_{1}}{K_{1}}+2 \Phi_{2}\left(\frac{l}{K_{2}}-\frac{l}{K_{1}}\right)+2 \frac{\rho \sigma}{R_{2}}\right)+4\left(\frac{\Phi_{2}}{K_{2}}+\frac{\rho}{2}\left(1-\frac{2 \sigma}{R_{2}}\right)\right)\right\}$

$$
+0\left(e^{-3}\right)
$$




$$
\bar{\Omega}_{1}=\frac{3 \sigma}{32 R_{2} \pi \mu_{0} \ell^{2}}[\bar{i} \times \bar{j}(\bar{j} \cdot \bar{i} \bar{i} \cdot \bar{F}+\bar{F} \cdot \bar{j})+\bar{i} \cdot \bar{j} \bar{F} \times \bar{j}]+0\left(e^{-3}\right),
$$

where $\rho$ is the distance from the center of hydrodynamic stress to the center of mass. The terms of $0\left(l^{-2}\right)$ in $(7.13)$ and (7.14) assume their greatest values when the force $\bar{F}$ is parallel to the wall. In fact when $\bar{F}, \bar{i}$ and $\bar{j}$ are parallel these terms vanish entirely. Two limiting cases will be considered when the force is parallel to the plane.

The first is Bretherton's [1962] ovoid of revolution which in polar coordinates is given by (Figure 1)

$$
r=a\left[1+\varepsilon P_{3}(\cos \theta)\right] \text {, }
$$

where $\varepsilon$ is a small parameter, and $\bar{j}$ is directed towards the "pointed" end. It is shown by Brenner [1964c] that for the ovoid the components of the resistance polyadics in $(7.13)$ and $(7.14)$ are

$$
\begin{gathered}
\frac{\Phi_{1}}{\mathrm{~K}_{1}}=\frac{5}{7} \varepsilon \mathrm{a}+O\left(\varepsilon^{2}\right), \frac{\Phi_{2}}{\mathrm{~K}_{2}}=-\frac{1}{7} \varepsilon a+O\left(\varepsilon^{2}\right), \\
\sigma-O\left(\varepsilon^{2}\right), \frac{1}{\mathrm{~K}_{1}}-\frac{1}{\mathrm{~K}_{2}}-O\left(\varepsilon^{2}\right), \rho-O(\varepsilon) .
\end{gathered}
$$

Hence from $(7.16)$ and $(7.17)$ it follows that to $O\left(\varepsilon^{2}\right)$ the ovoid will translate without rotation. The component of velocity parallel to the wall will be given correctly to $0\left(l^{-3}\right)$ by $(1.1 a)$; however, $(7.13)$ gives a velocity component perpendicular to the plane

$$
\bar{U}_{\perp}=\bar{i} \frac{3}{448} \frac{\varepsilon a}{\pi \mu_{0} \ell^{2}} \bar{F} \cdot \bar{j}
$$

Thus the ovoid will move respectively toward or away from the wall depending on whether it moves with the pointed or blunt end first (see Figure 1). 
For a body with fore-and-aft symmetry with its center of mass located at the center of symmetry the term of $0\left(e^{-2}\right)$ in $(7.13)$ is identically zero. The rotational motion is given by $(7.14)$, and if $\bar{j}$ and $\bar{F}$ are coplanar as shown in Figure $2 \mathrm{a}$ this reduces to

$$
\bar{\Omega}_{1}=\frac{3 \sigma F}{32 R_{2} \pi \mu_{0} \ell^{2}}\left[\sin ^{2} \xi-\cos ^{2} \xi\right] \bar{k},
$$

where $\bar{k}$ is a unit vector directed out of the plane of the paper and cos $\xi=$ $\bar{i} \cdot \bar{j}$ (see Figure 2a). When $\bar{j}$ and $\bar{F}$ are perpendicular $(7.14)$ reduces to

$$
\bar{\Omega}_{1}=-\frac{3 \sigma F \cos \theta}{32 R_{2} \pi \mu_{0} \ell^{2}} \bar{k} \text {. }
$$

where $\cos \theta=\bar{i} \cdot \bar{j}$ and $\bar{k}$ is directed out of the plane of the paper. Eqs. (7.19) and (7.20) are sufficient to determine the possible ultimate orientations of a body with fore-and-aft symmetry. The inner product of $\bar{F}$ and (7.14) easily yields the conditions for no rotation about an axis" parallel to $\overline{\mathrm{F}}$ to be $\in$ ither $\bar{k} \cdot \bar{i}=0$ or $\bar{F} \cdot \bar{j}=0$. Equations $(7.19)$ and $(7.20)$ then respectively give the conditions for no rotation as $\xi= \pm 45^{\circ}$ or $\pm 135^{\circ}$ and $\theta=90^{\circ}$.

For the circular disk $\sigma / R_{2}$ is $-\frac{1}{2}$, and from (7.19) it can be seen that when $\bar{k} \cdot \bar{i}=0$ it will rotate respectively clockwise or counterclockwise depending on whether $135^{\circ}>\xi>45^{\circ}$ or $45^{\circ}>\xi>-45^{\circ}$. All angles are included since $(7.14)$ is invariant to reflections of $\bar{j}$. Thus the ultimate motion of the disk is translation with its plane at $45^{\circ}$ to the wall, and because of this orientation the first two terms of (7.13) yield components of velocity directed away from the wall. If $\bar{j}$ and $\bar{F}$ are perpendicular and $\theta=\pi / 2$ then the disk moves without rotation parallel to the wall. 
b. The Cylinder

The cylinder is of general experimental interest because of its use in falling sphere viscometry. In this connection several authors (Tanner [1964], Wilson [1965]) have observed a marked tendency for spheres to migrate radially when they are launched along the centerline of a vertical tube. It is known from the Stokes flow analysis of Brenner and Happel [1958] that a sphere moving eccentrically in a tube experiences no sidewise force when the fluid is Newtonian. To extend their results to other geometries or to nonNewtonian fluids it is necessary only to insert the values of ${ }^{0}{ }_{I I}$ and $\nabla Q_{I I}$ for the cylinder into the appropriate equations of section 5 .

Cartesian coordinates $x_{1}, x_{2}, x_{3}$ are chosen with origin 0 located relative to the center of cylindrical symmetry $S$ by the constant vector $\bar{b}$, as shown in Figure 3. Relative to 0 the $x_{1}-x_{2}$ and $x_{1}-x_{3}$ planes are orthogonal planes of symmetry, and by conventional symmetry arguments the non-zero components of $\stackrel{\bar{Q}}{\mathrm{Q}}_{\mathrm{II}}$ and $\nabla \mathrm{Q}_{I I}$ are

$$
\begin{aligned}
& \mathrm{Q}_{\text {II11 }}^{\circ}, \mathrm{Q}_{\mathrm{II22}}^{\circ}, \mathrm{Q}_{\mathrm{II33}}^{\circ}, \\
& \partial_{1} 0_{I I I 1}^{0}, \partial_{2} Q_{I I 21}^{0}, \partial_{3}^{0} Q_{I I 3]}^{0}, \partial_{2} Q_{I I L 2}^{0}, \partial_{1} Q_{I I 22}^{0}, \partial_{3} Q_{I I I 3}^{0}, \partial_{1} Q_{I I 33}^{0} .
\end{aligned}
$$

The equation of continuity requires the sum of the first three terms in (7.2lb) to vanish, and hence $\bar{Q}_{\text {II }}^{\circ}$ has at most five independent components. At the present time $\overline{\bar{Q}}_{I I}\left(\bar{r}, \bar{r}^{\prime}\right)$ is not known for the cylinder ${ }^{8}$. Hence the components listed in (7.21) cannot be obtained by direct expansion of

8 Professon Brenner informs me that he and his students have obtained $\bar{Q}_{\text {II }}$ for the cylinder, and are presently evaluating some of its components. II 
$\overline{\bar{Q}}_{I I}$ as was done for the plane wall. Instead some of these components will. be back-calculated from certain results of the Newtonian analysis. Thus it will be necessary to consider two Taylor expansions, the first valid near the centerline and the second valid near the cylinder wall. Three orthogonal planes of symmetry intersect at $S$, the center of the cylinder, and consequently all the components of $\nabla \bar{Q}_{\text {II }}^{S}$ are zero. Thus the expansion (4.15) relative to $S$ is reduced to

$\overline{\bar{Q}}_{I I}=\overline{\bar{Q}}_{I I}+\frac{1}{2}\left(\overline{r r}: \nabla \nabla \bar{Q}_{I I}^{s}+\bar{r}^{\prime} \bar{r}: \nabla \nabla^{\prime} \overline{\bar{Q}}_{I I}^{s}+\overline{r r}^{\prime}: \nabla \nabla^{\prime} \overline{\bar{Q}}_{I I}+\bar{r}^{\prime} \bar{r}^{\prime}: \nabla^{\prime} \nabla^{\prime} \overline{\bar{Q}}_{I I}^{s}\right)+O\left(r^{4}\right)$.

The equation of continuity imposes the restrictions

$$
\nabla \nabla \cdot \overline{\bar{Q}}_{I I}^{s}=0, \quad \nabla^{\prime} \nabla \cdot \overline{\bar{Q}}_{I I}^{s}+\nabla^{\prime} \nabla \cdot \overline{\bar{Q}}_{I I}^{s}=0
$$

and the reciprocity relation $(4.5)$ requires

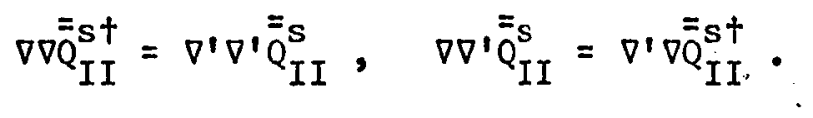

An expansion valid near the centerline is obtained by the transformation of the origin in (7.22) from $s$ to 0 . The radius vectors $\bar{r}_{0}$ and $\bar{r}_{0}^{\prime}$ are related to $\bar{r}$ and $\bar{r}^{\prime}$ respectively by

$$
\bar{r}=\bar{b}+\bar{r}_{0}, \quad \bar{r}^{\prime}=\bar{b}+\bar{r}_{0}^{\prime}
$$

The substitution of $(7.25)$ into $(7.22)$ results in a series in $\bar{r}_{0}$ and $\bar{r}_{0}^{\prime}$ of the form of $(4.15)$ with

$$
\begin{aligned}
& \overline{\bar{Q}}_{I I}=\overline{\bar{Q}}_{I I}^{s}+\frac{l}{2} \overline{b \bar{b}}:\left(\nabla \nabla \overline{\bar{Q}}_{I I}^{s \dagger}+\nabla \nabla \bar{Q}_{I I}^{s}+\nabla^{\prime} \nabla \overline{\bar{Q}}_{I I}^{s}+\nabla^{\prime} \nabla \bar{Q}_{I I}^{s \dagger}\right)+0\left(b^{4}\right), \\
& \nabla \overline{Q_{I I}}=\bar{b} \cdot \nabla \nabla \bar{Q}_{I I}^{s}+\frac{1}{2} \bar{b} \cdot\left(\nabla^{\prime} \nabla \bar{Q}_{I I}^{s}+\nabla^{\prime} \nabla \bar{Q}_{I I}^{s \dagger}\right)+o\left(b^{3}\right),
\end{aligned}
$$


Thus the leading terms in the centerline expansion are determined by $\overline{\mathrm{Q}}_{\mathrm{II}}$, $\nabla \nabla \bar{Q}_{I I}^{s}$ and $\nabla \cdot \nabla \bar{Q}_{I I}$. The leading terms in the expansion near the cylinder wall are deduced from the known values for the plane wall since the former approaches the latter as the radius $R$ becomes large. When in (7.3) $\ell$ and $\vec{i}$ are replaced by $R-b$ and $-\bar{i}_{1}$ respectively, and the results are expressed as the coefficients of a series in powers of $R^{-1}$ then the wall effect, tensors become

$$
\begin{aligned}
& \left.\bar{k}=\frac{9}{16} \frac{\left[\overline{\bar{l}}+\overline{\bar{i}}_{1} \bar{i}_{i}\right]}{(1-b / R)}+o\left(\frac{1}{1-b / R}\right)^{-1}, \bar{w} \sim \alpha \frac{1}{1-b / R}\right)^{2}, \\
& \overline{\bar{d}}=\frac{9}{32} \frac{\left[\bar{i}_{1} \bar{i}_{1} \bar{i}_{1}-\overline{\bar{i}_{1}}+\bar{i}_{1} \overline{\bar{l}}+\left(\bar{l} \bar{i}_{1}\right)^{\dagger}\right]}{(1-b / R)^{2}}+o\left(\frac{1}{1-b / R}\right)^{2} .
\end{aligned}
$$

This procedure for arriving at the limiting forms of the wall-effect tensors is justified, at least in part, by the analysis of Brenner and Happel [1958] who proved that $(7.28)$ holds rigorously for one of the components of $\bar{k}$.

In order to identify some of the components in (7.26) and (7.27) the translation of a sphere of radius a in a Newtonian fluid under the influence of a force $-\bar{F}_{3}$ is considered. From $(3.9),(3.10),(5.17),(5.18),(5.19)$ and (7.21) its velocity and constraint torque can be evaluated respectively as

$$
\begin{aligned}
& \bar{U}_{1}=-\frac{F}{6 \pi \mu_{0} a}\left(1-k_{33} \frac{a}{R}\right)^{\bar{i}_{3}}+0\left(\frac{a}{R}\right)^{3}, \\
& \bar{L}=-8 \pi \mu_{0} a^{2} w_{23}\left(\frac{a}{R}\right)^{2} U_{1} \bar{i}_{2}+0\left(\frac{a}{R}\right)^{3},
\end{aligned}
$$

where $\bar{L}$ is arrived at with $\bar{\Omega}_{1}=0$ in (5.18). From the Brenner-Happel results for this problem it is possible to identify $k_{33}$ and $w_{23}$ as

$$
k_{33}=f(b / R), \quad w_{23}=g(b / R)
$$


The function $f$ is tabulated in Happel and Brenner [1965] for the whole range of its argument. For small values of the argument $f$ and $g$ have the expansions

$$
\begin{aligned}
& f(b / R)=2.10444-0.6977(b / R)^{2}+0(b / R)^{4}, \\
& g(b / R)=1.296 b / R+0(b / R)^{3} .
\end{aligned}
$$

For values of $b / R$ near unity both $f$ and $g$ are in accord with (7.28). The 33-component of $\stackrel{0}{\mathrm{Q}}_{\mathrm{II}}$ can be written from $(7.26)$ as

$$
Q_{\text {II 33 }}^{0}=Q_{\text {II33 }}^{s}+b^{2}\left(\partial_{1} \partial_{1} Q_{\text {II33 }}^{s}+\partial_{1}^{\prime} \partial_{1} Q_{I I 33}^{s}\right)+0\left(b^{4}\right)
$$

It then follows directly from $(4.20),(7.31),(7.32 a)$ and $(7.33)$ that

$$
Q_{I I 33}^{s}=-2.1044(4 / 3 R), \partial_{1} \partial_{1} Q_{I I 33}^{s}+\partial_{1}^{\prime} \partial_{1} Q_{I I 33}^{s}=0.6977\left(4 / 3 R^{3}\right) .
$$

Likewise from (7.27) and (7.34) it follows that ${ }^{2}{ }_{1}{ }^{\circ}$ II 33 is given by

$$
\partial_{1} Q_{I I 33}^{0}=b\left(\partial_{1} \partial_{1} Q_{I I 33}^{s}+\partial_{1}^{\prime} \partial_{1} Q_{I I 33}^{s}\right)+0\left(b^{3}\right)=0.6977\left(\frac{4}{3} \frac{b}{R^{3}}\right)+0\left(b^{3}\right) .
$$

According to their definitions $(5.19 b) w_{23}$ and $d_{313}$ can be expressed in terms of $\partial_{1} Q_{I I 33}^{\circ}$ and $\partial_{3} Q_{I I I 3}^{\circ}$ by

$$
w_{23}=\frac{3 R^{2}}{8}\left(\partial_{1} Q_{I I 33}^{0}-\partial_{3} Q_{I I I 3}^{0}\right), d_{313}=-\frac{3 R^{2}}{8}\left(\partial_{3} Q_{I I I 3}^{0}+\partial_{1} Q_{I I 33}^{0}\right) \cdot(7.36)
$$

The component $d_{313}$ is conveniently expressible as

$$
d_{313}=h(b / R)
$$

where the function $h(x)$ has limiting values given by 


$$
\begin{aligned}
h(x) & =0.598 x+o\left(x^{3}\right) \text { as } x+0, \\
(1-x)^{2} h(x) & =9 / 32 \quad \text { as } x+1 .
\end{aligned}
$$

The expression for small $x$ is deduced from $(7.31),(7.32),(7.35)$ and for $x$ near unity from $(7.28)$. With these limiting values it is possible to construct a provisional plot for $h(x)$ for the whole range of $x$ similar to the one for $g(x)$ constructed by Happel and Brenner [1965] from similar limiting values.

The components $k_{33}, w_{23}, d_{313}$ are of interest to the purely Newtonian theory since with them it is possible to study the motion of particles of complex geometry. For instance, in an unbounded medium a uniform circular disk in free fall has no preferred orientation (see Happel and Brenner [1965]). However, at sufficiently low Reynolds numbers a disk falling near the axis of a cylinder will adopt the edgewise orientation. This phenomenon is readily explained by equation (5.18). Let $\bar{j}_{3}$ be a unit vector directed along the axis of the disk, and let $\bar{j}_{1}$ and $\bar{j}_{2}$ be orthogonal unit vectors in its plane. The angular velocity is then given by an expression similar to $(7.14)$ as

$$
\begin{aligned}
\bar{\Omega}_{1}=\left[\bar{i}_{2} w_{23}-\bar{j}_{2}\left\{\left(\bar{j}_{3} \cdot \bar{i}_{3}\right)\left(\bar{j}_{1} \cdot \bar{i}_{1}\right)+\left(\bar{j}_{1} \cdot \bar{i}_{3}\right)\left(\bar{j}_{3} \cdot \bar{i}_{1}\right)\right\} d_{313}\right. \\
\left.\quad+\bar{j}_{1}\left\{\left(\bar{j}_{3} \cdot \bar{i}_{3}\right)\left(\bar{j}_{2} \cdot \bar{i}_{1}\right)+\left(\bar{j}_{2} \cdot \bar{i}_{3}\right)\left(\bar{j}_{3} \cdot \bar{i}_{1}\right)\right\} d_{313}\right] \frac{F}{6 \pi \mu_{0} R^{2}} .
\end{aligned}
$$

The simplest case of interest occurs when $\bar{j}_{2}=\bar{i}_{2}$, and then (7.39) reduces to

$$
\bar{\Omega}_{1}=\bar{i}_{2}\left[g(b / R)+h(b / R)\left(\cos ^{2} \xi-\sin ^{2} \xi\right)\right] \frac{F}{6 \pi \mu_{0} R^{2}},
$$


where $\bar{j}_{3} \cdot \bar{i}_{1}=-\bar{j}_{1} \cdot \bar{i}_{3}=\cos \xi, \bar{j}_{3} \cdot \bar{i}_{3}=\bar{j}_{1} \cdot \bar{i}_{1}=\sin \xi$, and $\xi$ is the angle depicted in Figure 2a. From the limiting values of $g(7.32 b)$ and $h$ (7.38a) in the neighborhood of the centerline it can be seen that for all values of $\xi(7.40)$ predicts rotation which ultimately points the leading edge of the disk toward the centerline. In such an orientation the disk develops a sidewise velocity which carries it toward the centerline where its angular velocity is zero. If the leading edge continues across the centerline the rotation will be reversed as will the sidewise motion. Thus ultimately the disk will translate edgewise along the cylinder axis. The experiments of Squires and Squires [1937] indicate the edgewise orientation to be stable at Reynolds numbers below about 0.003 , while the planewise orientation is stable above 0.02. Cox [1965] has shown that the fluid's inertia tends to stabilize the planewise motion, but his analysis is valid only for an unbounded domain. Since (7.28) was derived from the plane wall results the disk will behave near the tube wall in the way already described in part 7a. That is, it will tend ultimately to orient itself with its plane at $45^{\circ}$ to the wall, and its leading edge directed away from it. Another special case of (7.39) occurs when the plane of the disk is in the 1-3 plane of the cylinder. The disk will then fall edgewise with an angular velocity about its own axis of $\bar{i}_{2}{ }_{23} F / 6 \pi \mu_{0} R^{2}$, and with no sidewise motion, which is the behavior which Brenner and Happel first demonstrated for the sphere.

In the application of the above results it should be borne in mind that their range of validity is confined to $a / R$ less than about 0.15 . For instance, Auerbach [1966] observed the motion of disks $(0.8>a / R>0.2)$ in a Newtonian fluid. When released near the axis in the planewise orientation the rotation of the disks was consistent with the predictions of $(7.40)$. However, 
instead of falling along the axis edgewise the disks performed a zig-zag motion between the axis and the wall. Auerbach also observed that when the disks were released edgewise they maintained their initial orientation which is in accord with (7.40).

As before the only particle which can be considered for non-Newtonian fluids is the sphere. The velocities of a sphere in free fall under the influence of an outside force $-\bar{F}_{3}$ can be calculated from (5.35) and (5.36) (the transient terms given by $(6.5)$ are negligible in this case)

$$
\begin{aligned}
& \bar{U}=-\bar{i}_{3}\left(U_{\infty}(F)-\frac{a}{R} k_{33} U_{\infty 1}\right)-\bar{i}_{1} \frac{a}{R^{2}}\left(\frac{\alpha_{1}+\alpha_{2}}{\mu_{0}}\right)\left(d_{313}-\frac{1}{2} d_{331}\right) U_{\infty 1}^{2},(7.41) . \\
& \bar{\Omega}=-\bar{i}_{2} \frac{a w_{23}}{l^{2}} U_{\infty 1}
\end{aligned}
$$

where the Stokes speed is $U_{\infty 1}$ is given by (3.9) as $F / 6 \pi \mu_{0} a_{\text {. It }}$ is easily verifiable from $(7.21)$ that there can be no other components of $\bar{U}$ or $\bar{\Omega}$. Each of the wall-effect tensor components in (7.41) and (7.42) is given above by approximate formulae valid near the centerline and the wall except for $d_{331}$ which has been determined only near the wall. However, it is easy to see from (7.21) that $d_{331}$ is of the form

$$
d_{331}=c \frac{b}{R}+o(b / R)^{3},
$$

where $c$ is an undetermined constant ${ }^{9}$. From (7.38a) and (7.41) it is clear that a calculation of this constant must give $\frac{1}{2} c>0.598$. Otherwise the analysis given in this paper will not explain the observed radial migration of spheres moving through polymer solutions.

\footnotetext{
9 It can be shown that $d_{331}$ can be calculated from a solution similar to that of Brenner and Happel ${ }^{31}$ for the case when the sphere moves along a chord of the cross section shown in Figure 3 .
} 
In falling sphere viscometry the terminal velocity is measured by timing a sphere over a known vertical distance. It is clear from (7.4I) that, in accurate work, measurement must also be made of the radial positions $b_{1}$ and $b_{2}$ of the sphere at the vertical positions $s_{1}$ and $s_{2}$ respectively. The vertical component of velocity is $\mathrm{ds} / \mathrm{dt}$, and by integration of (7.41) it is found from (7.32a) that

$$
\frac{s_{2}-s_{1}}{t}=U_{s}+0.6977 \frac{a}{R^{3}} \frac{U_{\infty 1}}{t} \int_{0}^{t} b^{2}(\theta) d \theta .
$$

where $t$ is the elapsed time, and

$$
U_{s}=U_{\infty}(F)-2.1044 \frac{a}{R} U_{\infty 1}
$$

The centerline velocity, $U_{S}$, is to $O\left(R^{-3}\right)$ the non-Newtonian equivalent of Faxen's wall correction formula. For a given sphere in a given tube the radial velocity $d b / d t$ near the centerline is from $(7.27)$ and $(7.4 i)$ of the form

$$
\frac{d b}{d t}=M b \text {, }
$$

and hence by integration $\ln \left(b_{2} / b_{1}\right)=M t$.

The constant $M$ can be deduced from (7.41) and will contain the unknown constant $c$ of $(7.43)$. With the help of (7.46) the integral in $(7.44)$ can be evaluated as

$$
\frac{1}{t} \int_{0}^{t} b(\theta)^{2} d \theta=\frac{b_{2}^{2}-b_{1}^{2}}{2 \ln \left(b_{2} / b_{1}\right)}
$$

where $M$ has been eliminated with $(7.47)$. From $(7.44)$ and by expansion of the 
logarithm in (7.48) the centerline velocity $U_{s}$ can be reduced to the formula

$$
u_{s}=\frac{s_{2}-s_{1}}{t}-0.6977 \frac{a}{R^{3}} U_{\infty 1}\left(\frac{b_{2}+b_{1}}{2}\right)\left[1-\frac{1}{6} \frac{\left(b_{1}-b_{2}\right)^{2}}{b_{2}\left(b_{2}+b_{1}\right)}+\ldots .\right] \text {. }
$$

This formula is sufficiently accurate for most practical purposes since terms of $O\left(b^{4}\right)$ in $(7.44)$ and $O\left(b^{3}\right)$ in $(7.46)$ have been neglected.

Tanner [1964] reports that radial migration increases with sphere size, and that beyond a certain size he observed a marked increase in the fall-time scatter. These results are in accord with $(7.41)$ and $(7.49)$. The Stokes speed is calculated from

$$
U_{\infty 1}=2 \Delta \rho a^{2} g / 9 \mu_{0},
$$

where $\Delta \rho$ is the density and the particle-fluid density difference and $g$ the gravitational acceleration. Hence according to $(7.41)$ the radial migration velocity increases as the fifth power of the sphere radius, and for the larger spheres the neglect of the second term in (7.49) will, manifest itself as scatter. Tanner also found the Faxen wall correction formula to be unsatisfactory. This is to be expected from (7.45) since for shear thinning fluids $U_{\infty}(F)$ is greater than $U_{\infty I}$; thus the usual Faxen formula overcorrects for the effect of the walls.

In addition to yielding a formula for the centerline velocity the migration velocity can be used to obtain an estimate of $\alpha_{1}+\alpha_{2}$ from radial position measurements. Near the wall $d_{313}$ and $d_{331}$ can be found from (7.28), and by integration the change in radial position is given by

$$
\left(R-b_{2}\right)^{3}-\left(R-b_{1}\right)^{3}=\frac{81}{64} \text { a } U_{\infty 1}^{2}\left(\frac{\alpha_{1}+\alpha_{2}}{\mu_{0}}\right) t \text {. }
$$

Hence if $\mu_{0}$ is known and $U_{\infty 1}$ is calculated from (7.50) then $\left(\alpha_{1}+\alpha_{2}\right)$ can be found. 
No terminal velocity data are available in which radial positions have been measured. Turian [1964] measured fall times for spheres in cylinders of seven different diameters. If the radial position correction is neglected these data provide a test of the centerline velocity formula (7.45) which is the one dimensional form of (1.1). In terms of the experimental quantities (7.45) becomes

$$
U=U_{\infty}-\frac{2.1044 \Delta \rho d^{3} g}{18 \mu_{0}} \frac{1}{D}\left(1-\frac{2.089}{2.104}\left(\frac{d}{D}\right)^{2}\right) \text {, }
$$

where $d$ and $D$ are respectively the sphere and cylinder diameters. Included in this formula is the next term in Faxen's series which provides an estimate of the neglected higher order terms. ${ }^{10}$ In the most extreme case the term in parenthesis modifies $2 / D$ by less than one per cent. In Figure 4 are shown typical plots of the terminal velocity data of Turian for a 1.25 per cent solution of hydroxyethyl cellulose (HEC) in water at $20^{\circ} \mathrm{C}$. Similar data for other sphere sizes were also obtained by Turian. From the least-square fitted lines, such as those shown in Figure 4 , values by $U_{\infty}$ and $\mu_{0}$ were calculated from (7.52). Since the effect of the cylinder wall in (7.52) is of the second order, accurate estimates of $\mu_{0}$ cannot be expected, and this is evident from the values in Table I. The large error estimates in $\mu_{0}$ arise because of the relatively smali change in terminal velocity over the range of cylinder sizes.

10 In a future publication it will be shown that the term of $O\left(D^{-3}\right)$ is indeed the next term in Faxen's series, and that the non-Newtonian effects are $O\left(F^{3} D^{-3}\right)$. 


\begin{tabular}{|c|c|c|c|c|}
\hline Sphere & diam. $\mathrm{cm}$ & $\mathrm{U}_{\infty} \mathrm{cm} / \mathrm{sec}$ & $\mu_{0}$ poise & $\Delta \rho d^{3} g m$ \\
\hline Ruby & 0.06350 & 0.00608 & $107+3$ & 0.000764 \\
\hline Ruby & 0.07620 & 0.00886 & $111 \overline{+2}$ & 0.00132 \\
\hline Ruby & 0.1000 & 0.0154 & $2: 29+10$ & 0.00298 \\
\hline Ruby & 0.15875 & 0.0410 & $108+2$ & 0.0119 \\
\hline Steel & 0.15875 & 0.108 & $96.7+6.7$ & 0.0272 \\
\hline
\end{tabular}

mean 110

Table I

For a given fluid the slope of the lines in Figure 4 depends on $\Delta \rho d^{3}$ which is shown in the last column of the table to change by a factor of almost 40 for these data. The terminal velocities of a steel sphere of twice the size of the one in the above table did not plot as a straight line. Upon addition of the contributions in (3.9) the sphere velocity in an unbounded region can be expressed as an apparent fluidity by

$$
\frac{6 \pi a U_{\infty}}{-F}=\frac{1}{\mu_{0}}-\frac{\lambda}{\mu_{0}^{3}}\left(\frac{F}{6 \pi a^{2}}\right)^{2}+0\left(\frac{F}{6 \pi a^{2}}\right)^{4},
$$

where $\lambda$ is a combination of material constants which can be found from (3.9). The apparent fluidity was calculated from the $U_{\infty}$ values in Table $I$, and a plot of this quantity against $\left(F / 6 \pi a^{2}\right)^{2}$ is shown in Figure 5. The four ruby spheres appear to fall into the range in which the term $0\left(F / 6 \pi a^{2}\right)^{4}$ is negligible as indicated by the close agreement of $\mu_{0}$ extrapolated from this plot and the mean value obtained from the wall effect. In fact the mean of the three best values in Table I is 109 Poise. Turian [1964] used an empirical method to obtain extrapolated values of $\mu_{0}$, and his value of 113 Poise is shown in Figure 5 for comparison. Turian has measured terminal velocities for other polymer solutions at various concentrations and temperatures, 
and similar treatment of these data gives the same degree of agreement between $\mu_{0}$ deduced from the wall effect and that found by extrapolation of the fluidity.

The value of $F / 6 \pi a^{2}$ for the steel ball is about five times that of the largest ruby sphere, and its apparent viscosity deviates significantly from the line of Figure 5. This behavior is in accord with the derivation of the wall effect formula (1.1) which remains valid beyond the range of the third order approximation to the Rivlin-Ericksen fluid from which (7.53) is derived.

The support of this work by the National Aeronautics and Space Administration under grants NsG-705 and NGR-40 is gratefully acknowledged. 


\section{Appendix}

Theories of the stress tensor involving memory functionals, usually integrals, can be shown to reduce to the approximations of the type given by $(2.4),(2.5)$ and $(2.6)$ by expansion of the strain tensor as a Taylor's series in powers of the time lapse. This process yields explicit formulae for the Rivlin-Ericksen coefficients in terms of the memory integrals, and since it is given elsewhere (see Truesdell \& Noll [1965]) in detail it will not be discussed further.

For theories of the differential type the series (3.1) and (3.2) are substituted into the constitutive equation, and upon collection of terms of the same order it is a matter of algebraic manipulation to show that the resulting stress approximations are precisely the ones obtained from the Rivlin-Ericksen approximations. This procedure will be illustrated for Oldroyd's [1958] model which can be written as

$$
\begin{aligned}
\overline{\bar{T}}+\lambda \frac{D \overline{\bar{T}}}{D t}-\mu_{1}\left(\overline{\bar{A}}(1) \cdot \overline{\bar{T}}+\overline{\bar{T}} \cdot \overline{\bar{A}}^{(1)}\right)+\frac{v_{1}}{2} \overline{\bar{I}} \overline{\bar{T}}: \overline{\bar{A}}^{(1)}+\frac{\mu_{0}^{\prime}}{2} \overline{\bar{A}}(1) \overline{\bar{I}}: \overline{\bar{T}} \\
\quad=n_{0}\left[\overline{\bar{A}}^{(1)}+\lambda_{2} \frac{D \overline{\bar{A}}(1)}{D t}-\mu_{2}\left(\overline{\bar{A}}^{(1)}\right)^{2}+\frac{v_{2}}{2} \overline{\bar{I}} \overline{\bar{A}}^{(1)}: \overline{\bar{A}}^{(1)}\right],
\end{aligned}
$$

where $\lambda, \mu_{1}, \nu_{1}, \mu_{0}^{\prime}, \lambda_{2}, \mu_{2}, \nu_{2}$, and $n_{0}$ are material constants. The stress $\overline{\mathrm{T}}$ is expanded as

$$
\overline{\bar{T}}=\overline{\bar{T}}_{1}+\overline{\overline{\mathrm{T}}}_{2}+\overline{\overline{\mathrm{T}}}_{3}+\cdots,
$$

and when (3.1), (3.2) and (A2) are substituted into (A1), and terms of the same order are collected the first two $\overline{\bar{T}}_{i}$ are found to be 


$$
\begin{aligned}
& \overline{\bar{T}}_{1}=-\overline{\bar{I}}_{p_{1}}+n_{0} \overline{\bar{A}}^{(1)}(1), \\
& \overline{\bar{T}}_{2}=-\overline{\bar{I}}_{p_{2}}+n_{0} \overline{\bar{A}}^{(1)}(2)+n_{0}\left(\lambda_{2}-\lambda_{1}\right) \frac{\partial \overline{\bar{A}}(1)}{D t}(1,1)+n_{0}\left(\mu_{1}-\mu_{2}\right)\left(\overline{\bar{A}}^{(1)}(1)\right)^{2},
\end{aligned}
$$

where the notation is the same as that used in (3.4) and (3.5). Comparison of (A3) and (A4) with the results obtained by the substitution of (3.1) and (3.2) into $(2.2),(2.4)$ and $(2.5)$ gives

$$
\begin{aligned}
& \mu_{0}=\eta_{0} \text { (zero-shear viscosity) } \\
& \alpha_{1}=\eta_{0}\left(\lambda_{2}-\lambda_{1}\right), \alpha_{1}+\alpha_{2}=\eta_{0}\left(\mu_{1}-\mu_{2}\right) .
\end{aligned}
$$

When $\overline{\bar{T}}_{3}$ is expressed similarly to $(A 3)$ and $(A 4)$ it can be shown that

$$
\begin{aligned}
\beta_{1}= & \eta_{0} \lambda_{1}\left(\lambda_{1}-\lambda_{2}\right), \beta_{2}+\beta_{3}=\frac{\eta_{0}}{2}\left[\left(\mu_{1}-\mu_{0}^{\prime}\right)\left(\mu_{1}-\mu_{2}\right)+\lambda_{1}\left(\lambda_{2}-\lambda_{1}\right)+\left(\mu_{1}-\frac{3}{2} \mu_{0}^{3}\right)\left(\nu_{2}-\nu_{1}\right)\right], \\
& \frac{3}{2} \beta_{1}+\beta_{2}=\eta_{0}\left[\mu_{1}\left(\lambda_{2}-\lambda_{1}\right)+\lambda_{1}\left(\mu_{2}-\mu_{1}\right)\right] .
\end{aligned}
$$

Spriggs [1966] has proposed a generalization of Oldroyd's model in. which the stress is constructed from a sum of contributions as

$$
\overline{\overline{\mathrm{T}}}=\sum_{n=1}^{\infty} \overline{\bar{T}}(n),
$$

where the $\overline{\bar{T}}(n)$ satisfy oldroyd's equation (Al) with material constants $\lambda_{1}^{(n)}, \lambda_{2}^{(n)}, \mu_{1}^{(n)}$, etc. These material constants are related to six independent constants $\lambda_{1}, \lambda_{2}, \varepsilon_{1}, \varepsilon_{2}, \alpha, n_{0}$ by $\lambda_{1}^{(n)}=\lambda_{1} n^{-\alpha}, \lambda_{2}^{(n)}=\lambda_{2}, \mu_{1}^{(n)}=\lambda_{1} n^{-\alpha}\left(1+\varepsilon_{1}\right)$, $\mu_{2}^{(n)}=\lambda_{2}\left(1+\varepsilon_{2}\right), \quad v_{1}^{(n)}=\frac{2}{3} \lambda_{1} n^{-\alpha}\left(1+\varepsilon_{1}\right), \quad v_{2}^{(n)}=\frac{2}{3}\left(1+\varepsilon_{2}\right) \lambda_{2}$,

$\mu_{0}^{(n)}=0, \quad n_{0}^{(n)}=n_{0} \frac{n^{-\alpha}}{Z(\alpha)}$, 
where $\mathrm{Z}($ ) is the Riemann zeta function

$$
z(x)=\sum_{n=1}^{\infty} n^{-x}
$$

The contributions $\overline{\bar{\tau}}(n)$ should not be confused with the terms in the perturbation series (A2). When the process carried out above for Qldroyd's equation is applied to the Sprigg's model the Rivlin-Ericksen coefficients can be shown to be given by

$$
\begin{aligned}
& \mu_{0}=\eta_{0}, \alpha_{1}=\eta_{0}\left(\lambda_{2}-\frac{Z(2 \alpha)}{Z(\alpha)} \lambda_{1}\right), \alpha_{1}+\alpha_{2}=\eta_{0}\left(\frac{Z(2 \alpha)}{Z(\alpha)} \lambda_{1}\left(1+\varepsilon_{1}\right)-\lambda_{2}\left(1+\varepsilon_{2}\right)\right), \\
& \beta_{1}=\frac{\eta_{0} \lambda_{1}}{Z(\alpha)}\left(Z(3 \alpha) \lambda_{1}-Z(2 \alpha) \lambda_{2}\right), \\
& B_{2}+B_{3}=\frac{\eta_{0}}{2 Z(\alpha)}\left[\frac{\lambda_{1}\left(1+\varepsilon_{1}\right)}{3}\left(Z(3 \alpha) \lambda_{1}\left(1+\varepsilon_{1}\right)-Z(2 \alpha)\left(1+\varepsilon_{2}\right) \lambda_{2}\right)+\lambda_{1}\left(Z(2 \alpha) \lambda_{2}-Z(3 \alpha) \lambda_{1}\right)\right], \\
& \frac{3}{2} \beta_{1}+\beta_{2}=\frac{\eta_{0}}{Z(\alpha)}\left[\lambda_{1}\left(1+\varepsilon_{1}\right)\left(Z(2 \alpha) \lambda_{2}-Z(3 \alpha) \lambda_{1}\right)+\lambda_{1}\left(Z(2 \alpha) \lambda_{2}\left(1+\varepsilon_{2}\right)-Z(3 \alpha) \lambda_{1}\left(1+\varepsilon_{1}\right)\right)\right]
\end{aligned}
$$

It should be bourne in mind that models such as 0ldroyd's and Spriggs' may give inadequate descriptions of the stress in certain flows. Hence Rivlin-Ericksen coefficients calculated from fitted values of the constants of these models may well be unrealistic.

Giesekus proposed a theory, equivalent to that of Rivlin and Ericksen, which is based on a set of kinematical tensors $\overline{(N)}$ constructed from

$$
\overline{\bar{f}}^{(1)}=\frac{1}{2} \overline{\bar{A}}^{(1)}, \quad \overline{\bar{f}}^{(N)}=\frac{D \overline{\bar{f}}(N-1)}{D t} \text {. }
$$


It follows from $(2.2)$ and $(A 12)$ that the $\overline{\bar{f}}(N)$ can be expressed directly in terms of the $\overline{\bar{A}}(N)$ and vice versa. For his third order approximation Giesekus [1963] writes

$$
\begin{aligned}
& \overline{\bar{T}}=-\overline{\bar{I}}_{p}+2 n_{0}\left[\overline{\bar{f}}^{(1)}+k_{0}^{(2)} \overline{\overline{\bar{f}}}(2)+k_{0}^{(11)}(\overline{\bar{f}}(1))^{2}+k_{0}^{(3)} \overline{\overline{\bar{f}}}(3)\right. \\
& \left.+k_{0}^{(21)}\left(\overline{\bar{f}}^{(2)} \cdot \overline{\bar{f}}^{(1)}+\overline{\bar{f}}^{(1)} \cdot \overline{\bar{f}}^{(2)}\right)+k_{l l}^{(1)} \overline{\bar{I}}: \overline{\bar{f}}^{(1)} \overline{\bar{f}}^{(1)}\right] .
\end{aligned}
$$

With the use of the identity (2.2) it follows from $(2.4),(2.5)$ and (2.6) that

$-\mu_{0}=n_{0}, \alpha_{1}=n_{0} k_{0}^{(2)},\left(\alpha_{1}+\alpha_{2}\right)=n_{0} k_{0}^{(11)} / 2, \beta_{1}=n_{0} k_{0}^{(3)}$,

$\beta_{2}+\beta_{3}=n_{0}\left(\frac{k_{1}^{(1)}}{4}-\frac{k_{0}^{(3)}}{2}\right), \frac{3}{2} \beta_{1}+\beta_{2}=n_{0} k_{0}^{(2 l)} / 2$.

The interrelationships between the above theories are particularly useful in checking results of perturbation calculations. For example, the quantity $\bar{U}_{\infty 33}$ in (3.9) was obtained from Giesekus [1963] in which he used his stress equation (Al3). The same problem had previously been solved by Leslie [1961] who used Oldroyd's equation and Caswell and Schwarz [1962] who used the Rivlin-Ericksen theory. With the identities given above, it is possible to show that the results for $\bar{U}_{\infty 3}$ of Leslie and Giesekus are in agreement while that of Caswell and Schwarz is probably in error.

The above mentioned theories describe isotropic fluids. In a series of papers Ericksen (see Truesdell \& Noll [1965]) has introduced and studied a class of anisotropic fluids. The behavior of these materials in slow flow has not been worked out in detail, but according to Denn and Metzner [1966] the theory appears to admit behavior different from that predicted by the Rivlin-Ericksen theory. 


\section{References}

Auerbach, J. M. [1966], Honors Dissertation, Division of Engineering, Brown University, Providence, R. I.

Brenner, H., and Happel, J. [1958], J. Fluid Mech., 4, 195.

Brenner, H. [1962], J. Fluid Mech., 12, 35-48.

Brenner, H. [1964a], J. Fluid Mech., 18, 144-158.

Brenner, H. [1964b], Chemical Engrg. Sci., 19, 599-629.

Brenner, H. [1964c], Chemical Engrg. Sci., 19, 631-651.

Bretherton, F. P. [1962], J. Fluid Mech., 14, 284-304.

Caswell, B., and Schwarz, W. H. [1962], J. Fluid Mech., 13, 417-426.

Coleman, B. D., and Walter Noll [1961], Ann. New York Acad. Sci., 89, 672-714.

Cox, R. G. [1965], J. Fluid Mech., 23, 273.

Denn, M. M. and Metzner, A. B. [1966], Trans. Soc. of Rheology, 10, 215.

Giesekus, Hanswalter [1963], Rheologica Acta, 3, 59-71.

Green, A. E., and Rivlin, R. S. [1960], Arch. Rat. Mech. Anal., 4, 387.

Happel, John \& Howard Brenner [1965], "Low Reynolds Number Hydrodynamics,"

Prentice-Hall, Inc., Englewood Cliffs, N. J.

Langlois, W. E., and Rivlin, R. S. [1959], "Steady Flow of Slightly Viscoelastic Fluids," Tech. Rept. No. 3, Division of Applied Math., Brown University, Providence, R. I.

Leslie, F. M. [1961], Quart. J. Mech. and Applied Math., 14, 36-48.

Oldroyd, J. G. [1958], Proc. Roy. Soc. (Londca), A245, 278.

Rivlin, R. S., and Ericksen, J. L. [1955], J. Rat'l. Mech, and Anal., 4, $350-362$. 


\section{References (continued)}

Spriggs, T. W. [1966], Ph.D. Dissertation, Department of Chemical Engineering, University of Wisconsin, Madison, Wisconsin.

Squires, L., and W. Squires, Jr. [1937], Trans. Amer. Inst. Chem. Engrs., 33, 1 .

Tanner, R. I. [1964], Chem. Engrg. Sci., 19, 349-355.

Truesdell, C. \& W. Noll [1965], "The Non-Linear Theories of Mechanichs, Hanbuch der Physik, Vol. III/3, S. Flügge, Editor, Berlin-HeidelbergNew York: Springer.

Turian, R. M. [1964], Ph.D. Dissertation, Department of Chemical Engineering, University of Wisconsin, Madison, Wisconsin.

Williams, W. E. [1966], J. Fluid Mech., 24, 285-291.

Wilson, D. S. [1965], M.S. Dissertation, Department of Chemical Engineering, University of California (Davis).

edf

jb 


\section{Captions for Figures}

Figure 1. Motion of an Ovoid with the Gravity Force Parallel to a Plane.

Figure 2. Rotation of a Disk Near a Plane.

a. Diameter Perpendicular to the Gravity Force.

b. Diameter Parallel to the Gravity Force.

Figure 3. Cartesian Coordinates for the Cylinder.

The $x_{3}$-axis is directed out of the page.

Figure 4. The Effect of Cylinder Size on the Terminal Velocity of Spheres. Data of R. M. Turian [1964] for 1.25\% HEC in Water.

Figure 5. Fluidity Extrapolation Plot for the Data of R. M. Turian [1964]. 


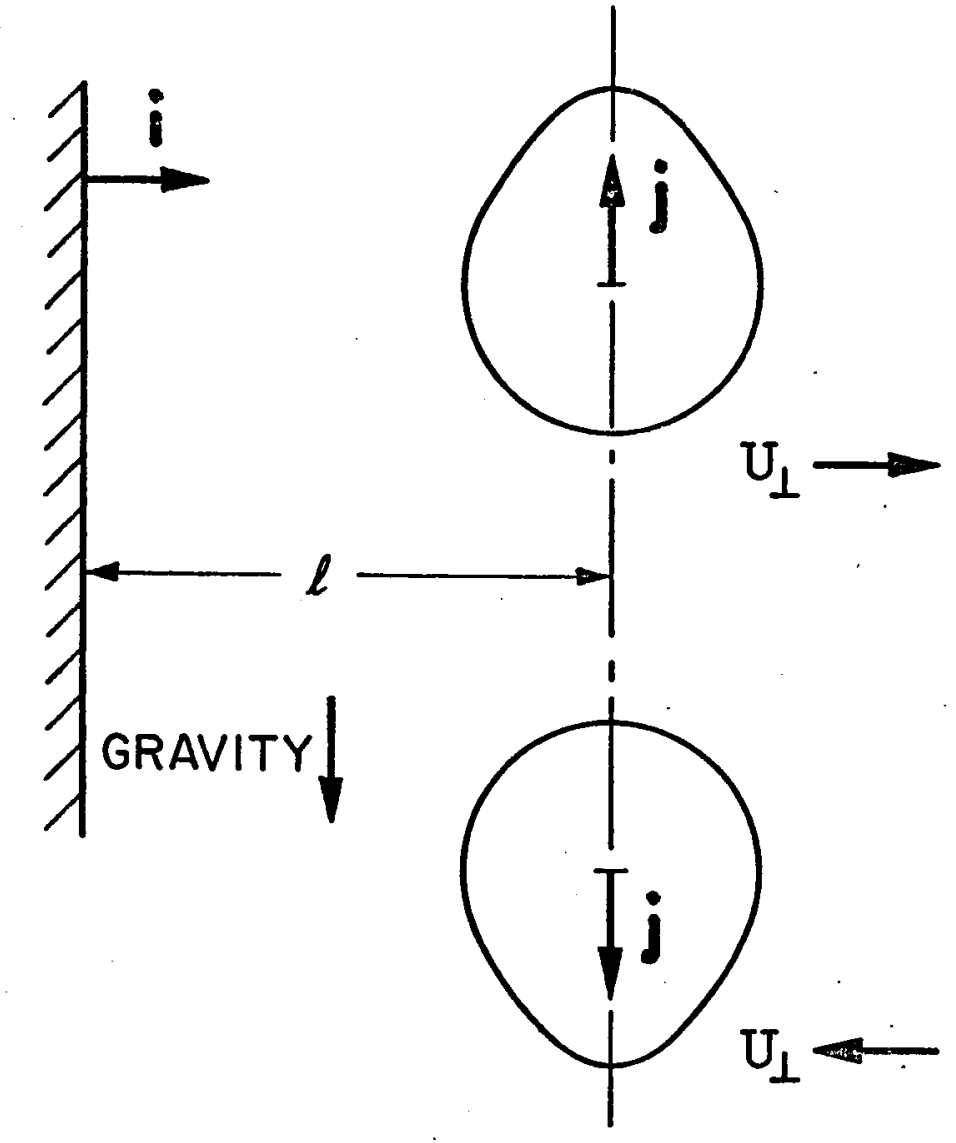

FIGURE I

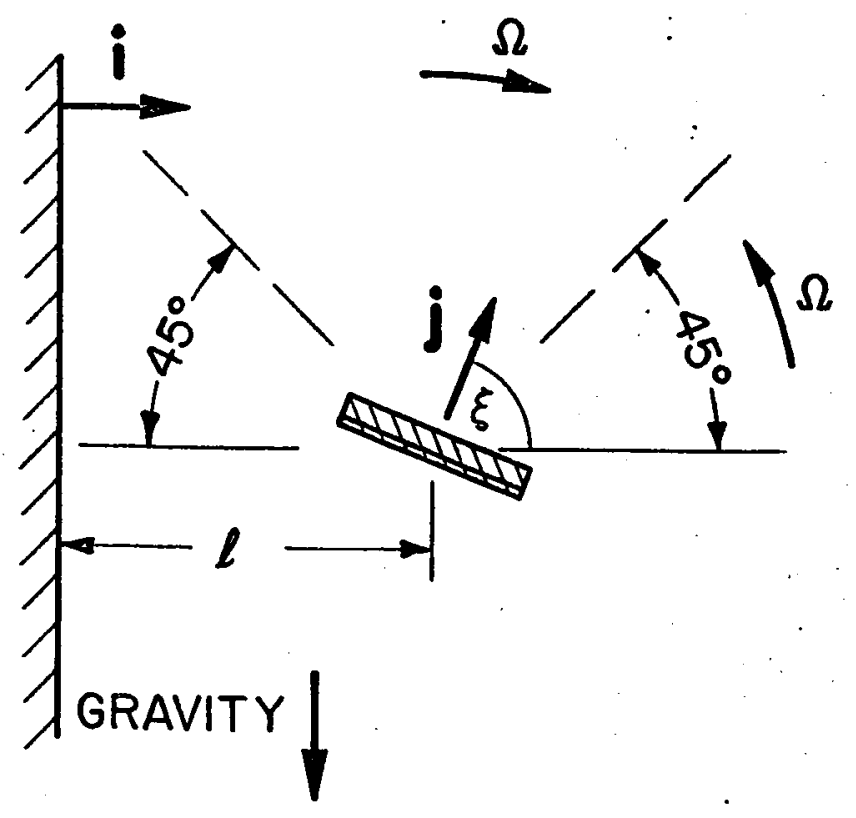

a

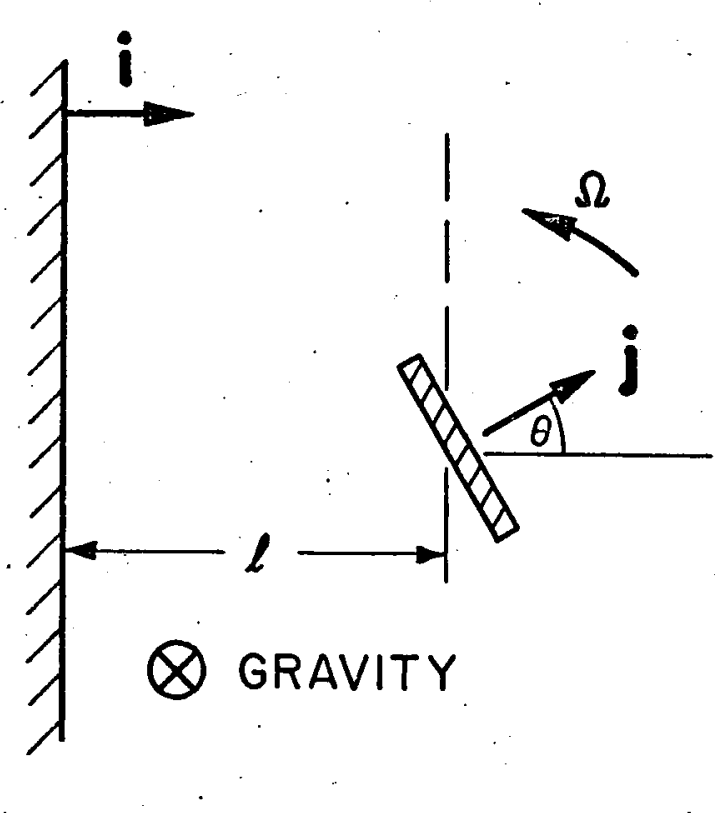

b

FIGURE 2 

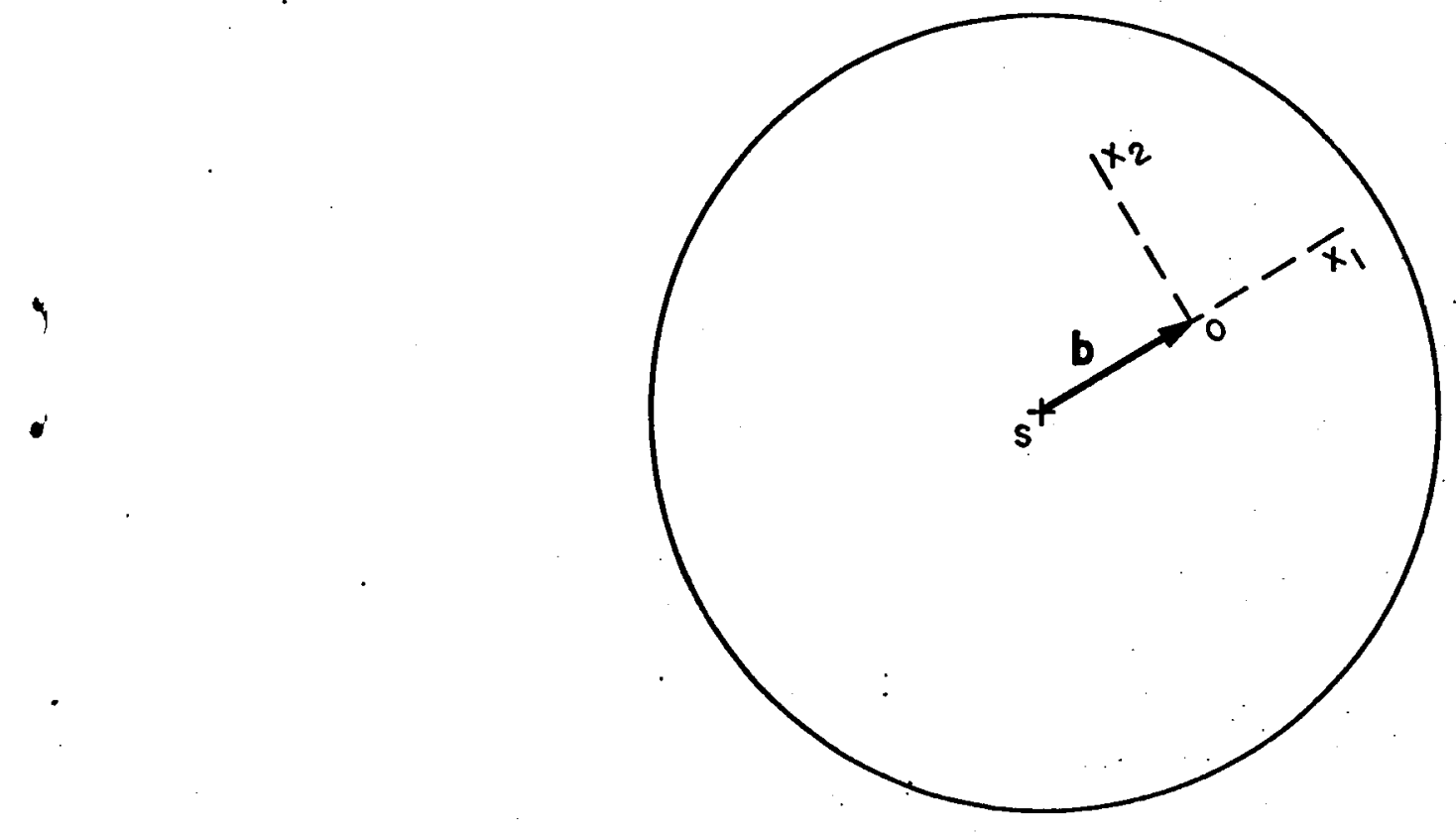

FIGURE 3 


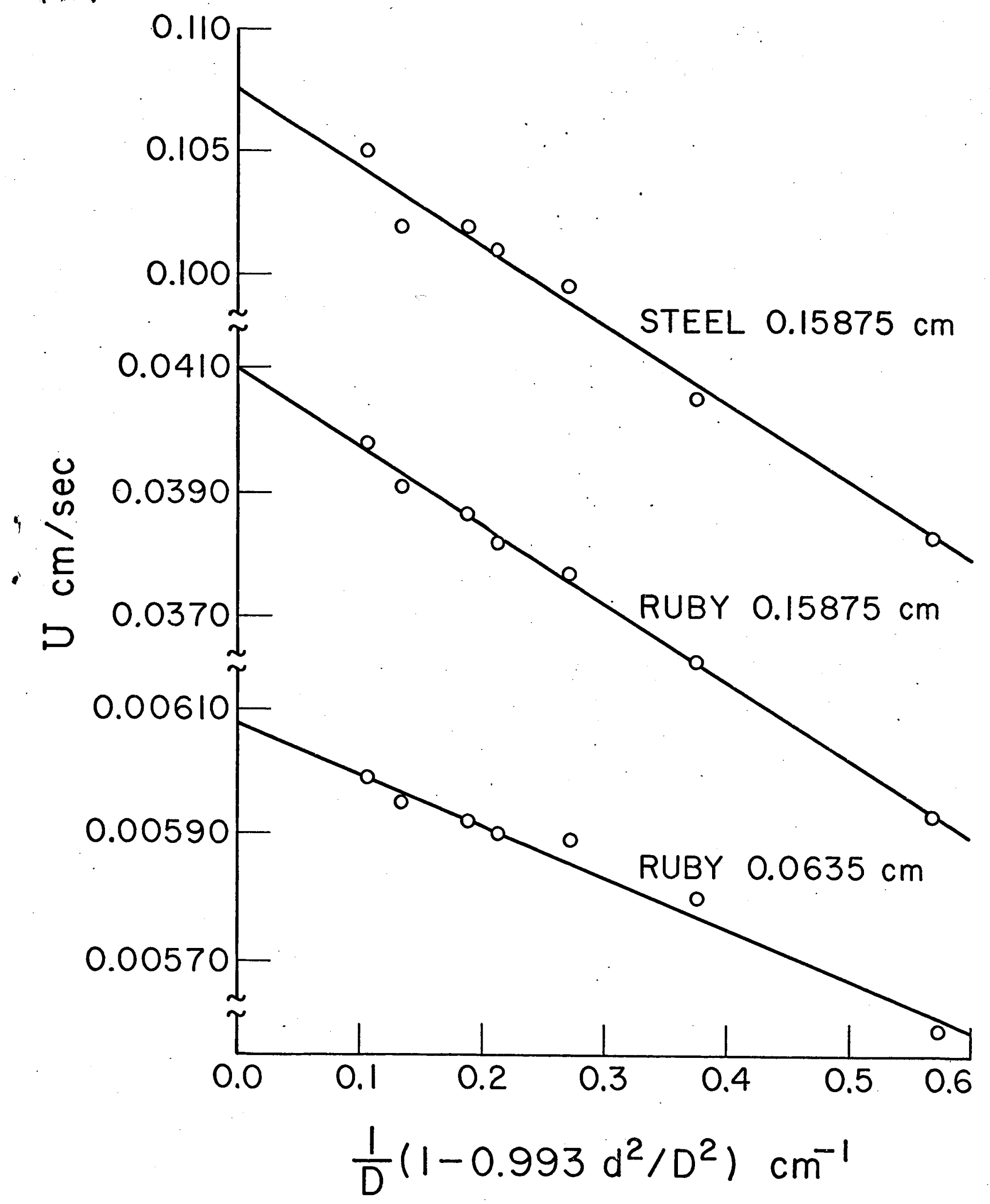

FIGURE 4 


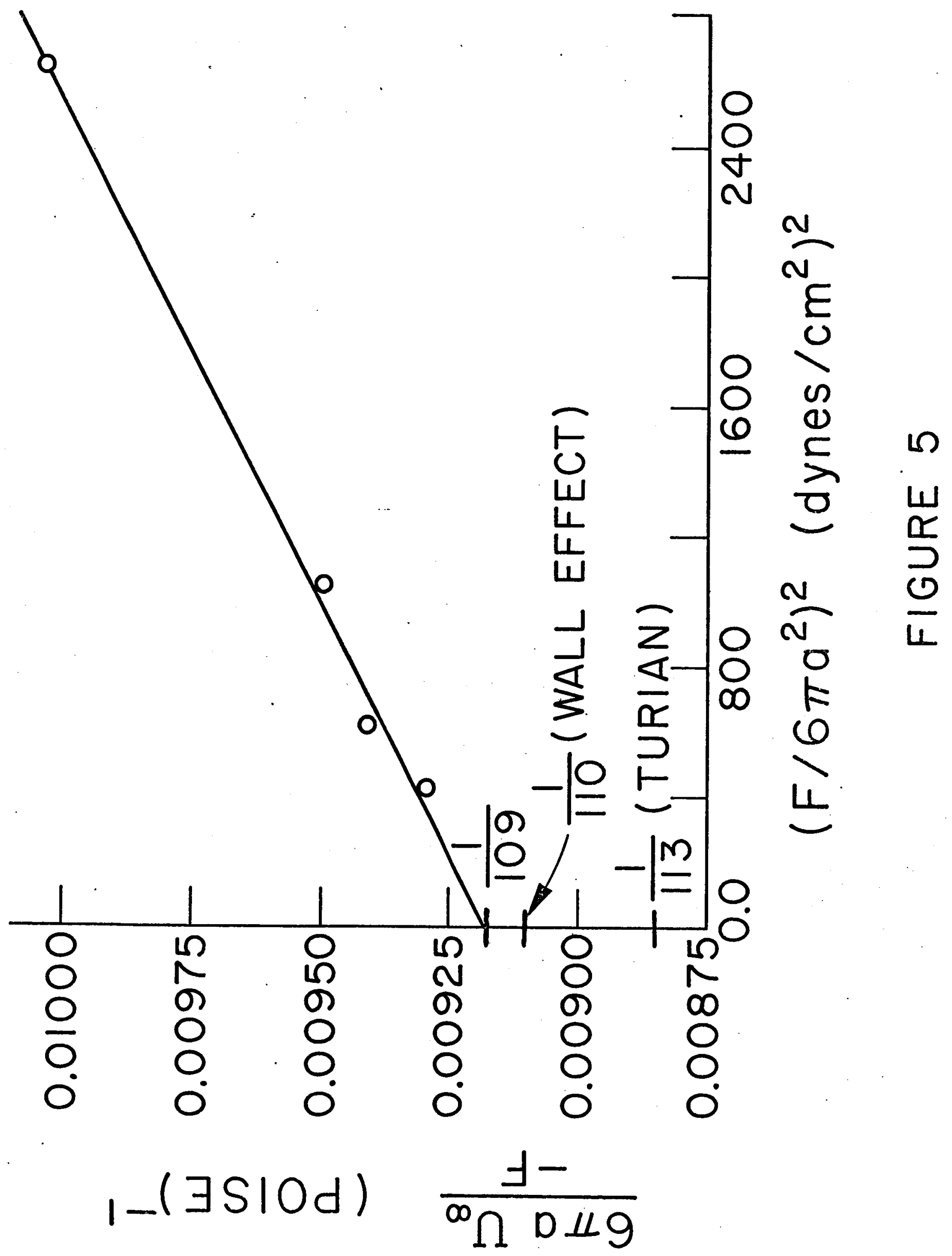

